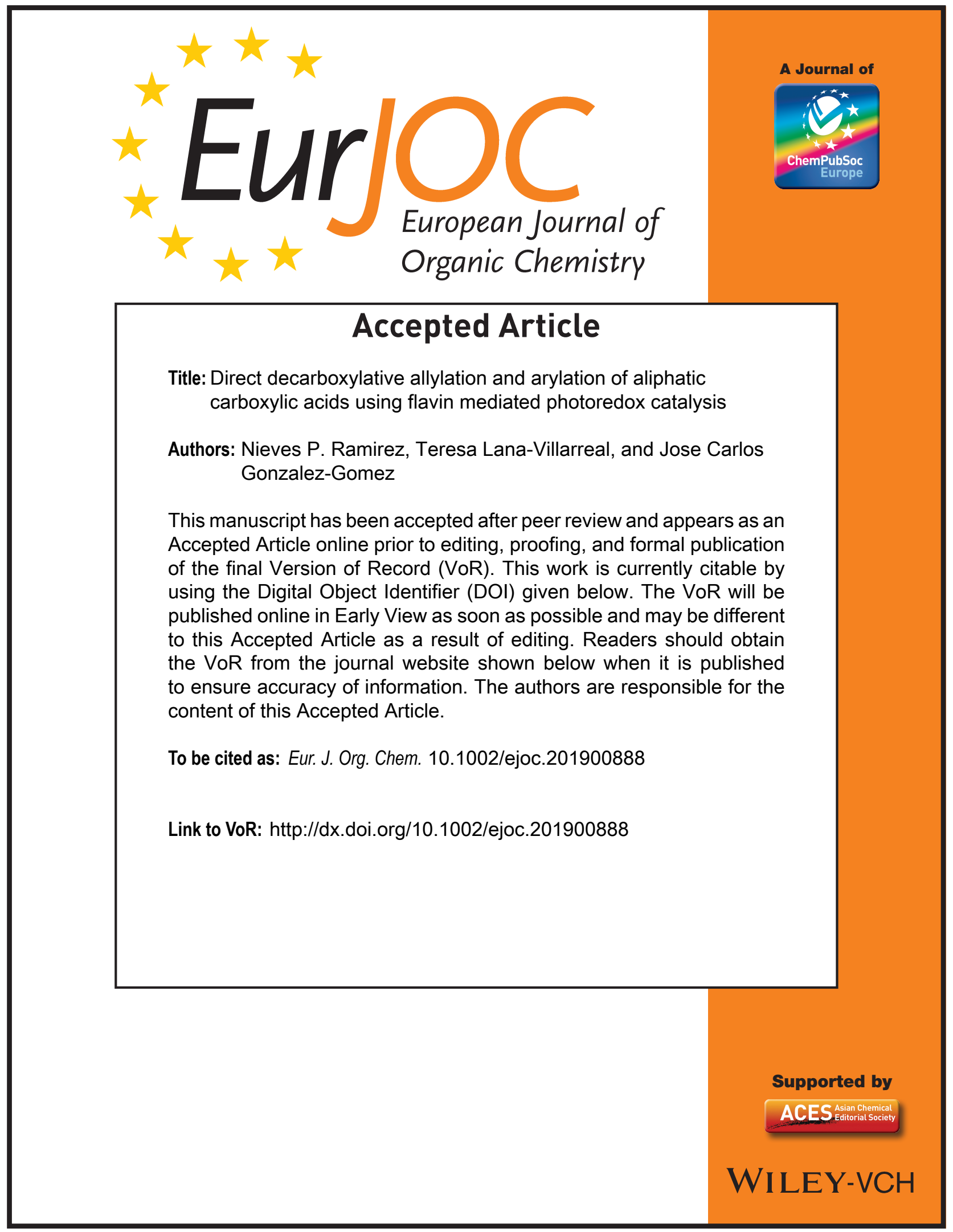




\title{
Direct decarboxylative allylation and arylation of aliphatic carboxylic acids using flavin mediated photoredox catalysis
}

\begin{abstract}
Nieves P. Ramirez, ${ }^{[a]}$ Teresa Lana-Villarreal, ${ }^{[b]}$ and Jose C. Gonzalez-Gomez ${ }^{*[a]}$
Abstract: We describe herein a direct decarboxylative allylation of aliphatic carboxylic acids with allylsulfones using visible light and riboflavin tetraacetate (RFTA) as photocatalyst. The reaction proceeds at room temperature tolerating a wide range of functionalities, avoiding the use of external bases or additives. Mechanistic studies support that alkyl radicals are involved in the reaction, and that a true photocatalytic cycle is operating. It is proposed that the carboxylic acid is deprotonated by [RFTA $]^{-}$, and the corresponding carboxylate acts as reductive quencher of RFTA $^{*}$, which after decarboxylation produces the alkyl radical. The methodology was adapted to prepare benzothiazoles substituted at C2, by reacting some carboxylic acids with 2-(phenylsulfonyl)benzothiazole. The number of carboxylic acids suitable for this arylation was lower than for the allylation and this different reactivity was briefly commented.
\end{abstract}

\section{Introduction}

In recent years, photoredox catalysis has made important progresses on the use of visible light energy to activate organic molecules and promote their reactivity under very mild and sustainable conditions. ${ }^{[1]}$ In this context, the photocatalytic oxidation of aliphatic carboxylic acids is a very convenient approach to generate free radicals, after fast decarboxylation of the corresponding acyloxy radicals $\left(\mathrm{k} \sim 10^{9} \mathrm{~s}^{-1}\right.$ at $\left.25{ }^{\circ} \mathrm{C}\right) .^{\left[{ }^{[2]}\right.}$ Although other radical precursors have also been successfully exploited (e.g. alkyltrifluoroborates ${ }^{[3]}$ silicates,${ }^{[4]}$ dihydropyridines, ${ }^{[5]}$ and Katritzky pyridinium salts $\left.{ }^{[6]}\right)$, carboxylic acids are ideal starting materials because they are abundant, inexpensive, stable and some of them are derived from biomass. ${ }^{[7]}$ The vast majority of photocatalytic decarboxylative functionalizations of carboxylic acids use bases in stoichiometric amounts to facilitate the oxidation of the corresponding carboxylate. This is not surprising if we consider that the oxidation potential of the carboxylic acid (e.g. for phenylacetic acid $E_{1 / 2}\left(\mathrm{RCO}_{2} \mathrm{H}^{+} / \mathrm{RCO}_{2} \mathrm{H}\right)=+2.51 \mathrm{~V}$ vs SCE$)$ is much higher than the one corresponding to the carboxylate (for tetrabutylammonium phenylacetate: $E_{1 / 2}\left(\mathrm{RCO}_{2}{ }^{\circ} / \mathrm{RCO}_{2}{ }^{-}\right)=+1.27 \mathrm{~V}$ vs. SCE $) .{ }^{[8]}$ Actually, functionalized carboxylic acids have been used in a variety of photocatalytic oxidative transformations of other functional groups relying on the fact that the carboxyl group is not oxidizable itself. ${ }^{[9]}$ In practice, the photocatalytic oxidation of carboxylic acids to acyloxy radicals usually involves a deprotonation by an external base, followed by a photoinduced electron transfer (PET) to strongly oxidizing photocatalysts (Scheme 1a). Although some iridium photocatalysts have very favorable redox potentials (e.g.

[a] M.Sc. N. P. Ramirez, Prof. J. C. Gonzalez-Gomez Instituto de Síntesis Orgánica y Departamento de Química Orgánica Universidad de Alicante

Apdo 99, E-03080 Alicante, Spain

E-mail: josecarlos.gonzalez@ua.es http://iso.ua.es

[b] Prof. T. Lana-Villarreal

Instituto de Electroquímica y Departamento de Química Física Universidad de Alicante

Supporting information for this article is given via a link at the end of the document. for $\left[\operatorname{Ir}\left(\mathrm{dF}\left(\mathrm{CF}_{3}\right) \mathrm{ppy}\right)_{2}(\mathrm{bpy})\right] \mathrm{PF}_{6}: E_{1 / 2}\left({ }^{*} \mid \mathrm{r}^{\prime \prime \prime} / / \mathrm{r}^{\prime \prime}\right)=+1.32 \mathrm{~V}$ vs. SCE $)^{[10]}$ for this transformation and convenient long excited state lifetimes, they are very rare and expensive, being organic dyes inexpensive and environmentally friendly alternatives.

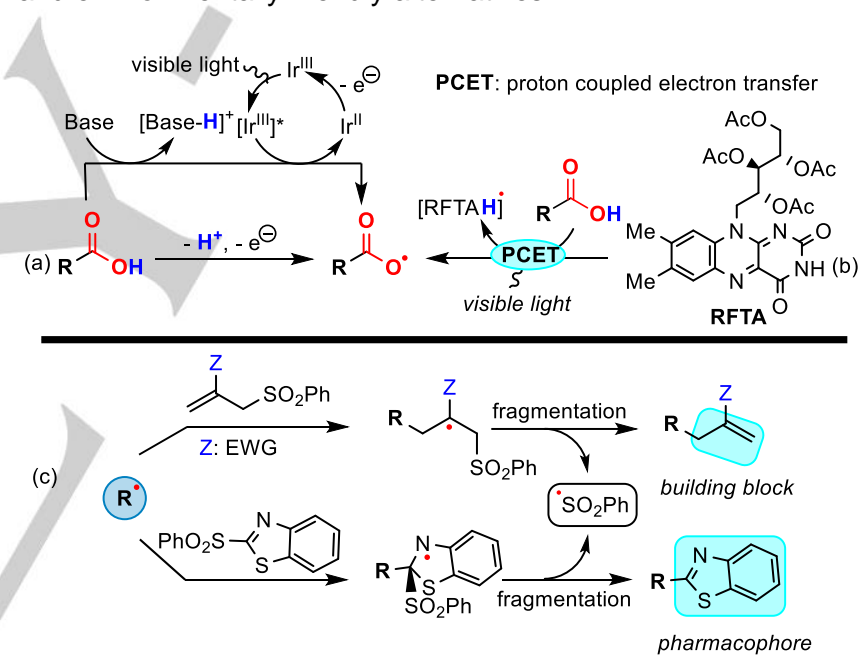

Scheme 1. (a) Photocatalytic oxidation of carboxylic acids with $\mid \mathrm{r}^{\mathrm{III}}$-derived photocatalyst. (b) RFTA acting as base and photocatalyst in the photooxidation of carboxylic acids. (c) Radical addition to allylsulfones or to 2(phenylsulfonyl)benzothiazole, followed by elimination of phenyl sulfonyl radical.

Among different oxidizing organic photocatalysts, we were particularly attracted by riboflavin (RF), ${ }^{[11]}$ a natural compound also known as vitamin $B_{2}$, which is very abundant and responsible for the redox activity of several flavo-enzymes. ${ }^{[12]}$ Upon visible light irradiation ( $\lambda_{\max } \sim 450 \mathrm{~nm}$ ), RF is excited to its short-lived singlet state $\left({ }^{*} S_{1}, \tau=6.8 \mathrm{~ns}\right)$ and undergoes an efficient intersystem crossing $\left(\phi_{\mathrm{ISC}}=0.38\right)$ to obtain a long-lived triplet state $\left({ }^{\star} T_{1}, \tau=15 \mu \mathrm{s}\right)$, which is oxidant enough $\left(E_{1 / 2}\left({ }^{3} \mathrm{RF}^{*} / \mathrm{RF}^{*}\right)=\right.$ $+1.50 \mathrm{~V}$ vs SCE) to oxidize carboxylates salts $\left(+1.2 \mathrm{~V} \leq E_{1 / 2} \leq\right.$ $+1.5 \mathrm{~V}$ vs SCE). ${ }^{[13]}$ Notably, Gilmour's group reported that photoexcited RF is able to oxidize cinnamic acids and biaryl carboxylic acids, in the absence of any external base, to generate the corresponding carboxyl radical. ${ }^{[14]}$ It was proposed that the 
flavin moiety $\left(\mathrm{p} K_{\mathrm{a}}\right.$ of $\mathrm{RFH}^{\cdot}=8.3$ in $\mathrm{H}_{2} \mathrm{O}$ ) can deprotonate the aliphatic carboxylic acid $\left(\mathrm{p} K_{a} \sim 5\right.$ in $\mathrm{H}_{2} \mathrm{O}$ ), before the PET, or in a formal proton-coupled electron transfer (PCET). In line with these results, MacMillan's group reported that riboflavin tetrabutyrate photocatalyzes the decarboxylative alkylation of peptides at the C-terminal carboxylic acid site more efficiently under mildly acidic conditions ( $\mathrm{pH}$ 3.5). ${ }^{[15]}$ Inspired by these findings, we have developed very recently a protocol in which a riboflavin tetraacetate (RFTA) acts as base and photocatalyst in the decarboxylative cyanation of aliphatic carboxylic acids, avoiding the use of stoichiometric bases. ${ }^{[16]}$ Thus, we decided to extend the application of this economic, user-friendly and sustainable protocol to generate alkyl radicals (Scheme 1b) and explore other organic transformations. It is known that nucleophilic alkyl radicals are rapidly added to electron-deficient alkenes, generating stable radical intermediates, that can easily eliminate a sulfonyl radical from the $\beta$ position (Scheme 1c). ${ }^{[17]}$ In this context, we planned to examine the direct decarboxylative allylation of aliphatic carboxylic acids with allylsulfones using visible light and RFTA without any other additive. Our interest on this radical allylation stems from the fact that both substrates- carboxylic acids and allyl sulfones- are readily available, and the resulting alkene could serve as a versatile precursor by synthetic manipulations of the allyl moiety. Moreover, we also decided to examine 2(phenylsulfonyl)benzothiazole as radical acceptor to obtain 2alkyl benzothiazole derivatives, ${ }^{[18]}$ an heterocyclic family with multiple bioactivities. ${ }^{[19]}$

A fast and chemoselective photocatalytic decarboxylative allylation was recently developed (Scheme 2a), but using $\mathrm{N}$ acyloxyphthalimides, $\left[\mathrm{Ru}(\mathrm{bpy})_{3}\right]\left(\mathrm{PF}_{6}\right)_{2}$ as photocatalyst and stoichiometric amounts of reductants. ${ }^{[20]}$ The silver catalyzed direct decarboxylative allylation of carboxylic acids (Scheme $\mathbf{2 b}$ ) was also recently developed, showing broad substrate scope, but in this case $\mathrm{K}_{2} \mathrm{~S}_{2} \mathrm{O}_{8}$ was required in stoichiometric amounts to regenerate the $\mathrm{Ag}(\mathrm{II})$ catalyst. ${ }^{[21]}$ The direct photocatalytic allylation of carboxylic acids via a redox-neutral process was not developed until very recently using $\operatorname{Ir}(\mathrm{ppy})_{2}(\mathrm{bpy}) \mathrm{PF}_{6}$ as catalyst, and stoichiometric amounts of $\mathrm{Cs}_{2}\left(\mathrm{CO}_{3}\right)$, although it was limited to $\mathrm{N}$-arylglycine derivatives (Scheme 2c). ${ }^{[22]}$ Herein we report our results on the direct and redox-neutral photocatalytic allylation of carboxylic acids (Scheme 2d).

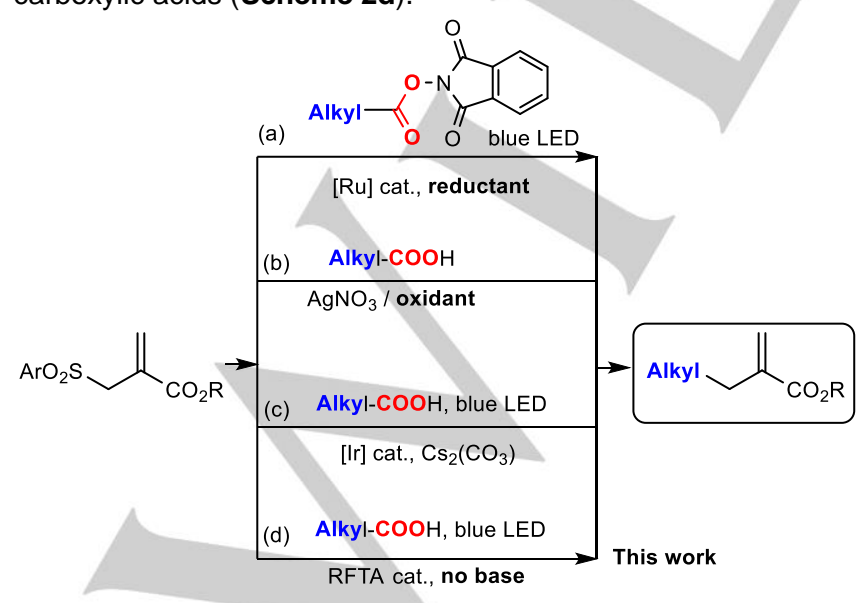

Scheme 2. Precedents in the photocatalytic decarboxylative allylation of carboxylic acids with allyl sulfones.

\section{Results and Discussion}

As in our previous communication, we have chosen RFTA ${ }^{[23]}$ as the photocatalyst for this study because it shows better solubility in organic solvents, greater photostability and is less likely than $\mathrm{RF}$ to form aggregates through hydrogen bonding interactions. ${ }^{[24]}$ A key feature in our redox-neutral visible light-promoted decarboxylative allylation of carboxylic acids with allylsulfones is the turnover of the RFTA catalyst with the phenyl sulfonyl radical $\left(\mathrm{PhSO}_{2}{ }^{\circ}\right)$. In our reaction design, (Scheme 3) a formal protoncoupled electron transfer between the photoexcited RFTA* and the carboxylic acid would explain the generation of alkyl radicals in the absence of base. Alternatively, it is plausible that a small fraction of in situ formed [RFTA] ${ }^{-}$deprotonates the carboxylic acid to give the corresponding carboxylate, ${ }^{[25]}$ which in turn would be oxidized by the long-lived triplet-excited state of the flavin, generating the alkyl radical after rapid decarboxylation. ${ }^{[26]}$ The addition of alkyl radicals to electron-deficient allylsulfones should be relatively fast $\left(k \sim 10^{6} \mathrm{M}^{-1} \mathrm{~s}^{-1}\right),{ }^{[20]}$ and must be followed by a radical fragmentation to obtain the desired alkene. In the same event is produced the stabilized phenyl sulfonyl radical, which is easily reduced $\left[E_{1 / 2}\left(\mathrm{PhSO}_{2} / \mathrm{PhSO}_{2}{ }^{-}\right)=+0.50 \mathrm{~V} \text { vs SCE}\right]^{[27]}$ by the hydroflavin radical $\left[E_{1 / 2}\left(\mathrm{RFTA}_{\mathrm{RFTAH}}\right) \approx-0.60 \mathrm{~V}\right.$ vs SCE], ${ }^{[28]}$ thereby closing the catalytic cycle after proton transfer. According to the redox potentials involved, this electron transfer is highly exergonic $\left(\Delta G_{E T} \approx-25 \mathrm{kcal} / \mathrm{mol}\right)$. However, we can not rule out a hydrogen atom abstraction by phenyl sulfonyl radical from the hydroflavin radical to complete the photoredox neutral catalytic cycle.

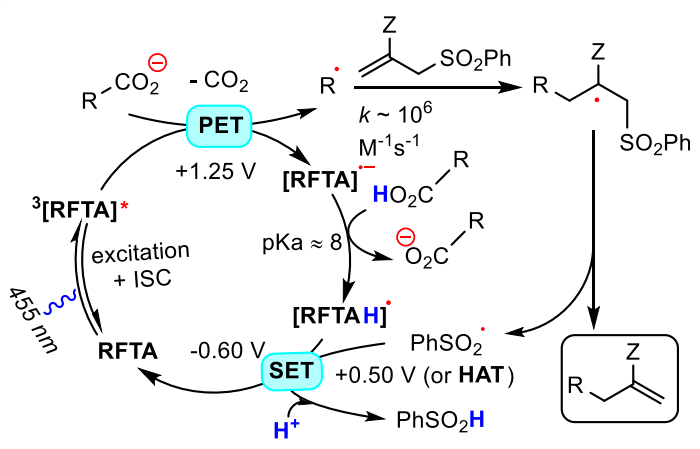

PET: Photoinduced Electron Transfer

SET: Single Electron Transfer

HAT: Hydrogen Atom Transfer

Scheme 3. Plausible mechanism for our photocatalytic decarboxylative allylation of carboxylic acids.

Our studies began using 2-phenoxypropanoic acid (1a) and butyl 2-((phenylsulfonyl)methyl)acrylate (2a) as model substrates to screen different conditions for the decarboxylative allylation reaction (Table 1). When the reaction was performed using degassed MeCN (argon sparging over $10 \mathrm{~min}$ ) and RFTA (5 mol-\%) as photocatalyst, under irradiation with blue LEDs $\left(\lambda_{\max }\right.$ 
$455 \mathrm{~nm}, 15 \pm 2 \mathrm{~mW} / \mathrm{cm}^{2}$ ) at room temperature, the desired product 3aa was obtained in $77 \%$ yield after $22 \mathrm{~h}$ (entry 1). Notably, using a higher load of RFTA (entry 2) did not provide any significant advantage over the previous conditions. Moreover, other solvent systems (entries 3 and 4) or the addition of an organic base (entry 5) in stoichiometric amount had a deleterious effect on the product yield. Incomplete conversion was observed when the reaction was run for only $8 \mathrm{~h}$ (entry 6 ). Furthermore, when the reaction was not degassed, product 3aa was also obtained in a diminished yield (entry 7). Finally, control experiments revealed that both the light and the photocatalyst are required to the reaction takes place (entries 8 and 9). It is worth of note that although the allyl sulfone is frequently used in large excess ( $3-4$ equiv) to minimize other competing reactions, we have optimized our reaction using an almost perfect stoichiometry.

Table 1. Optimization of the decarboxylative allylation.

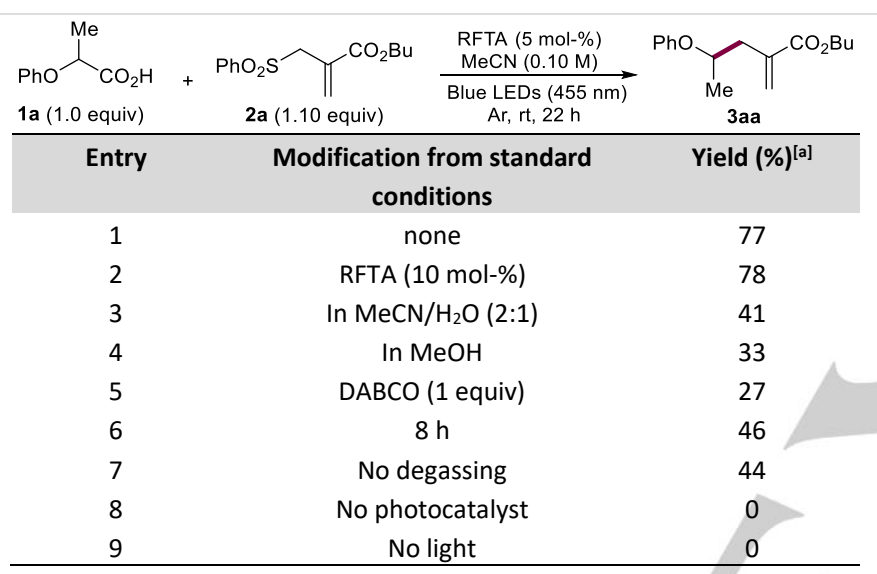

[a] Yield determined by GC using adamantane as internal standard.

Under our optimized reaction conditions, we examined different carboxylic acids with allylsulfone 2a (Scheme 4). We were pleased to observe that different $\alpha$-oxo carboxylic acids- including secondary, tertiary and even primary- were suitable substrates, giving the desired products (3aa-3ia) from moderate to good yields. Interestingly, the ribosic acid derivative afforded the desired product 3 ha in good yield as a single isomer with retention of the configuration, ${ }^{[29]}$ although a longer time and two portions of $5 \mathrm{~mol}-\%$ of photocatalyst were required. Moreover, an $\alpha$ benzyloxy carboxylic acid was also tolerated with the catalytic protocol, although the corresponding product 3ia was obtained in relatively low yield. Remarkably, an $\alpha$-thio- carboxylic acid was also compatible under the reaction conditions, giving the desired product $\mathbf{3 j a}$ in good yield, despite different possible parasitic reactions (e.g. overoxidation, homocoupling of intermediate radicals, hydrodecarboxylation, etc). In addition, an $\alpha$-ketoacid was also a suitable substrate affording the desired ketone $\mathbf{3 k a}$ in a moderate yield. $\mathrm{N}$-arylglycine derivatives were also well tolerated under the optimized conditions and the corresponding products (3la-3oa) were obtained from moderate to good yields. Similarly, a pyrrole-derived carboxylic acid afforded the desired product $3 \mathrm{pa}$ in good yield. Unfortunately, other common $\mathrm{N}$ protecting groups, such as Boc and $\mathrm{Cbz}$, were not suitable to transform the alanine derivatives in useful synthetic yields. We also explored a few non-activated $\alpha-C$ carboxylic acids, but only the tertiary adamantly carboxylic acid gave product 3 qa in moderate yield. The cyclohexyl- and sec-butyl carboxylic acids failed to give the desired products in significant amounts.

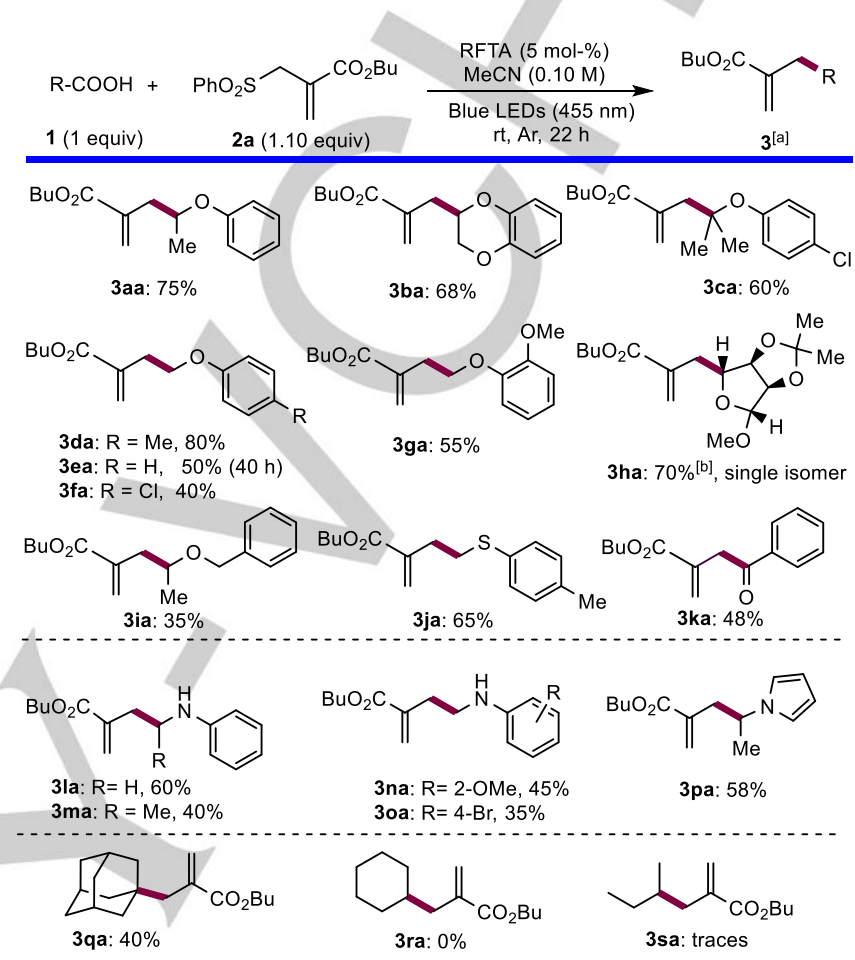

Scheme 4. Scope of carboxylic acids on the decarboxylative allylation. [a] Yields are reported for isolated pure products at $0.25 \mathrm{mmol}$ scale. [b] After $24 \mathrm{~h}$, another $5 \mathrm{~mol}-\%$ of RFTA was added and the reaction was run over $12 \mathrm{~h}$.

We also examined the scope of different allylsulfones under our optimized conditions, using 2-phenoxypropanoic acid as model substrate (Scheme 5). Terminal allylsulfones with different ester groups, including ethyl, benzyl, and allyl esters were compatible with this protocol, giving the corresponding products (3ab-3ad) in good yields. Other electron-withdrawing groups were also well tolerated in the terminal allylsulfones, such as chloro and phenylsulfone, giving the desired compounds 3ae and 3af in good yields. In addition, an electron-richer 2-phenylallylsulfone was also a suitable partner, likely by resonance stabilization of the benzylic radical intermediate, to provide product 3 ag in a moderate yield. Moreover, internal allyl sulfone $\mathbf{2} \mathbf{h}$ was also tested, obtaining the terminal alkene 3ah in low yield after a difficult purification, as a 2:1 mixture of diastereoisomers. This result supports a regioselective alkyl radical addition to the double bond of the allyl sulfone, followed by $\beta$-elimination of the sulfonyl radical $\left(S_{\mathrm{H}} 2\right.$ ' pathway). Remarkably, the mild reaction conditions used were compatible with a broad range of functionalities, including: esters, ethers, phenyl rings, chloro substituents, ketals, thioethers, ketones, $\mathrm{N}$-aryl amines, pyrroles, allyl groups, benzyl groups, sulfones and cyano groups. 


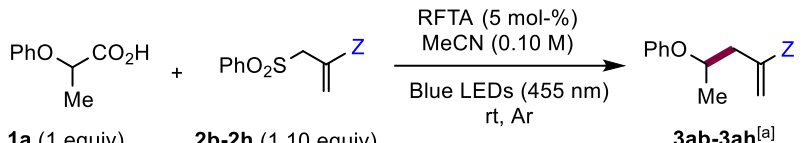

$$
\begin{aligned}
& \text { 1a (1 equiv) 2b-2h (1.10 equiv) } \quad \text { 3ab-3ah }{ }^{[a]}
\end{aligned}
$$

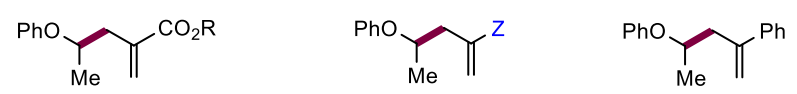

$$
\begin{aligned}
& \text { 3ab: } R=E t, 76 \%(12 h) \\
& \text { 3ac: } R=B n, 65 \%(12 \mathrm{~h}) \\
& \text { 3ad: } R=\text { allyl, } 65 \%(12 \mathrm{~h})
\end{aligned}
$$

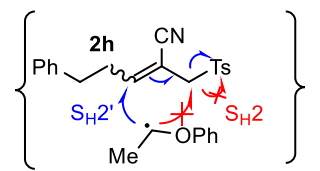

$$
\begin{aligned}
& \text { 3ae: } Z=C l, 60 \%(12 h) \\
& \text { 3af: } Z=\mathrm{SO}_{2} \mathrm{Ph}, 68 \%(40 \mathrm{~h})^{[\mathrm{b}]} \\
& \text { 3ag: } 40 \%(22 \mathrm{~h}) \\
& \mathrm{CN} \\
& \mathrm{OPh}_{\mathrm{OPh}}^{\mathrm{CN}} \\
& \text { 3ah: } 32 \%(2: 1 \mathrm{dr})^{[\mathrm{c}]}, 48 \mathrm{~h}^{[\mathrm{b}]}
\end{aligned}
$$

Scheme 5. Allyl sulfone scope with carboxylic acid 1a. [a] Yields are reported for isolated pure products at $0.25 \mathrm{mmol}$ scale. [b] 2 equiv of 1a were used. [c] Determined by ${ }^{1} \mathrm{H}-\mathrm{NMR}$.

To examine the synthetic utility of our direct decarboxylative allylation, we carried out the preparation of compound 3 ba at 2 $\mathrm{mmol}$ scale under batch conditions (Scheme 6). Although the product was obtained in a synthetically useful yield (48\%), a significant decreased was observed compared to the reaction at $0.25 \mathrm{mmol}(68 \%) .{ }^{[30]}$ Moreover, to show that the allyl moiety can also serve as a radical acceptor, we conducted the Giese decarboxylative alkylation using reaction conditions previously developed in our group. ${ }^{[31]}$ Under these conditions, when carboxylic acid 1d was employed, compound 6 was obtained in an unoptimized $45 \%$ yield.

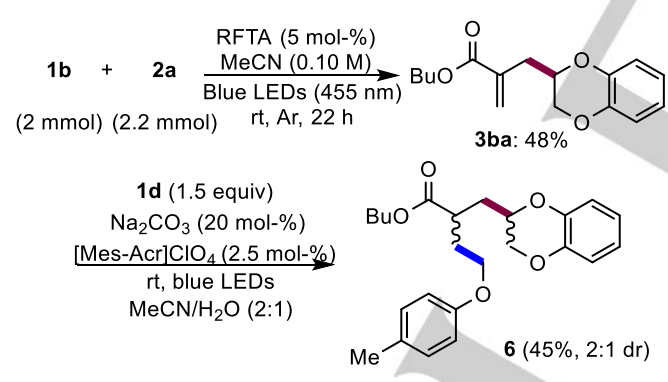

Scheme 6. Preparation of 3 ba at $2 \mathrm{mmol}$ scale and Giese decarboxylative alkylation with carboxylic acid $\mathbf{1 d}$.

Evidence for the free radical involvement in these reactions follows from the observed inhibitory effect of TEMPO $((2,2,6,6$ tetramethylpiperidin-1-yl)oxyl, Scheme 7a) on the allylation reaction. Eventually, the corresponding radical was trapped by TEMPO on this experiment to form adduct 1b-TEMPO, which was detected by LC-MS. Additionally, when carboxylic acid 1q was submitted to the allylation conditions with sulfone $\mathbf{2 a}$, the expected compound 3oa was isolated, but also compound 7 from a fast 5 exo-trig radical addition followed by the allylation reaction (Scheme 7b). In order to support the proposed mechanism, it was also checked that carboxylic acid $\mathbf{1 b}$ was an efficient quencher of RFTA* luminescence's, while the emission intensity of the photoexcited catalyst was not altered in the presence of $\mathbf{2 a}$ (Scheme 7c, details can be found in the supporting information). Finally, the quantum yield of the reaction of $\mathbf{1} \mathbf{b}$ and $\mathbf{2 a}$ resulted very low (Scheme 7d), supporting a closed photocatalytic cycle instead of a radical chain propagation. ${ }^{[32]}$

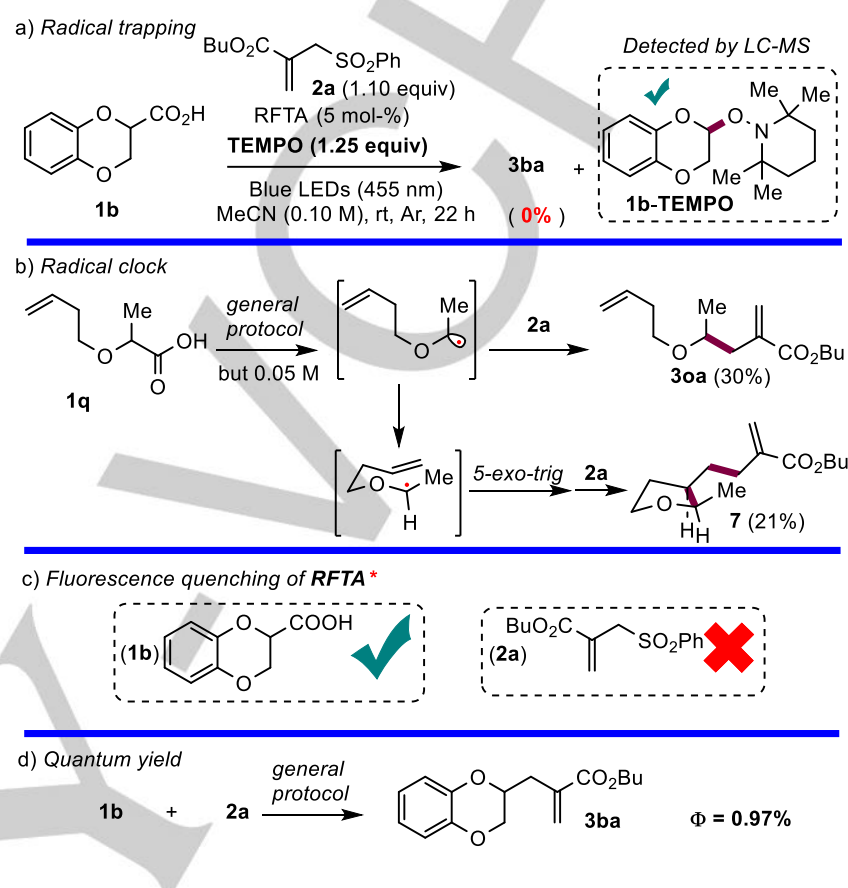

Scheme 7. Mechanistic studies.

We thus re-examined the reaction conditions to adapt our protoco for the decarboxylative arylation of aliphatic carboxylic acids, using $\mathbf{1 b}$ and 2-(phenylsulfonyl)benzothiazole (4) as substrates (Table 2). Under the same allylation conditions the desired compound $\mathbf{5 b}$ was obtained in moderate yield (entry 1 ). When 2 equivalents of the carboxylic acid were used, the reaction yield was slightly improved (entry 2). Different solvent systems were also examined to facilitate the solubility of the aryl sulfone at the beginning of the reaction, but none of them furnished the desired product in a better yield (entries 3-5). Finally, when the load of photocatalyst was increased to $10 \mathrm{~mol}-\%$ and the reaction was run over $36 \mathrm{~h}$, the best result was obtained (entry 6). Notably, this reaction is slower than the allylation, likely by a higher steric demand in the addition of the alkyl radical to the carbon atom next to the bulky sulfonyl group. It is worth saying that we recognized that a possible solution to this problem would be the C2-alkylation of unsubstituted benzothiazoles, thereby less sterically hindered and more easily available starting materials. ${ }^{[33]}$ However, the reaction of $\mathbf{1 b}$ with benzothiazole, using RFTA (10 mol-\%) and air as terminal oxidant gave only traces of product $\mathbf{5 b}$ (not shown). 
Table 2. Re-examining reaction conditions for the decarboxylative arylation.

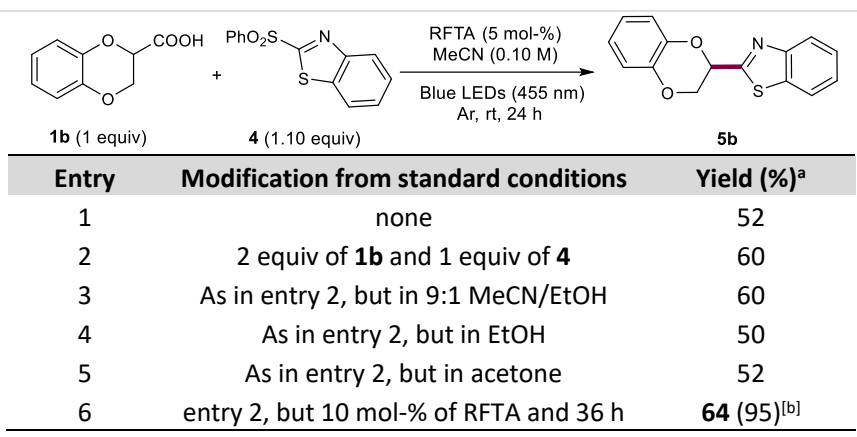

[a] Yields determined for isolated pure products. ${ }^{[b]}$ Based on recovered compound 4.

Having identified reasonably good reaction conditions, we screened some aliphatic carboxylic acids in the homolytic aromatic substitution (HAS) of 2-(phenylsulfonyl)benzothiazole (Scheme 8). As in the decarboxylative allylation, $\alpha$-oxo carboxylic acids were suitable substrates in this transformation, obtaining the products $\mathbf{5 a - 5 e}$ in yields from moderate to good. It is worth noting that during the formation of compound $\mathbf{5 d}$ it was also observed the homocoupling of the corresponding radicals by GCMS. In the case of compound $\mathbf{5 e}$ the yield was also rather low, most likely due to a higher steric hindrance with the bulky radical involved in the transformation. Finally, the $\mathrm{C} 2$ - $\alpha$-aminomethylation of benzothiazole was accomplished using $\mathrm{N}$-phenylglycine in our reaction conditions to obtain $\mathbf{5} \mathbf{f}$ in good yield. Other carboxylic acids, such as: clofibric acid (1c), 2-(p-tolylthio)acetic acid (1j) and phenoxyacetic acid (1e) failed to give the desired benzothiazole, observing mainly the corresponding hydrodecarboxylation by GCMS. Moreover, N-Boc phenylalanine gave only traces of the desired benzothiazole.
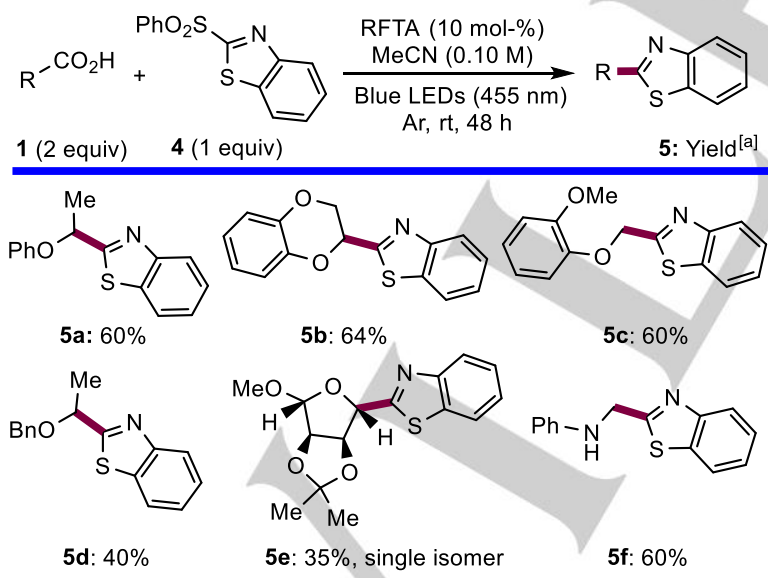

Scheme 8. Carboxylic acids scope in the HAS of 4. [a] Yields are reported for isolated pure products at $0.25 \mathrm{mmol}$ scale.

In our previous decarboxylative cyanation with TsCN, using RFTA as base and photocatalyst, the range of successful carboxylic acids was broader, being $\alpha-N$ carbamates and non-activated $\alpha-C$ carboxylic acids also suitable substrates. ${ }^{[16]}$ In this work, we have observed that $\alpha$-thio-, tertiary $\alpha$-oxo- and $\alpha$-keto carboxylic acids were successful in the decarboxylative allylation, but not in the arylation reaction. It is evident that most successful carboxylic acids in these reactions have an $\alpha$-substituent that stabilizes the radical by resonance, thereby increasing the energy of the SOMO and their reactivity with electron-deficient radical acceptors. From Kochi's work it is known that decarboxylation $\left(k_{d e c}\right)$ of arylacetoxy radicals generated via PET in carboxylate ion pairs lies in the range of $10^{9} s^{-1}$, competing with very fast back-electron transfer $\left(\mathrm{K}_{\mathrm{BET}} \sim \mathbf{1 0}^{\mathbf{1 1}} \boldsymbol{s}^{\mathbf{- 1}}\right){ }^{\left[{ }^{2]}\right.}$ In this study was also concluded that decarboxylation of $\alpha$-oxo benzylic carboxylic acids to provide stabilized benziloxy radicals is extremely fast $\left(\mathrm{k}_{\mathrm{dec}} \sim \mathbf{1 0}^{\mathbf{1 1}} \boldsymbol{s}^{\mathbf{- 1}}\right)$. We thus reasoned that the success of these reactions depends on an extremely fast decarboxylation to override the backelectron transfer. It is also important the use of very electrophilic radical acceptors (with low steric demand) that rapidly trap the generated radical (in very low concentration), whereas the sulfonyl radical is produced to regenerate the RFTA and reset a new catalytic cycle. From the simplified picture depicted in Scheme 9 it is also possible to anticipate that, if the reaction of the generated radical is not fast $\left(\mathrm{k}_{\mathrm{R} 1}\right.$ or $\left.\mathrm{k}_{\mathrm{R} 2}\right)$, the hydrogen atom abstraction from RFTAH would be possible (hydrodecarboxylation) or even deactivation of the catalyst by radical-radical coupling.

$$
\begin{aligned}
& \left.\begin{array}{ccc}
\mathrm{X}: \mathrm{NAr}, \mathrm{SAr} \\
\mathrm{O}
\end{array}\right]
\end{aligned}
$$

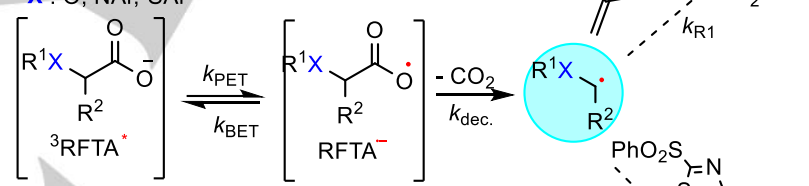

$$
\begin{aligned}
& \because \because{ }_{R 2} \\
& \text { ( }
\end{aligned}
$$

Scheme 9. Different reactivity of carboxylic acids with the radical acceptors used.

\section{Conclusions}

In conclusion, we have demonstrated that the direct decarboxylative allylation of carboxylic acids with allylsulfones can be efficiently promoted at room temperature by visible light, using inexpensive RFTA as photocatalyst in the absence of base. The transformation is redox-neutral, avoiding the use of external additives and none of the reagents was used in large excess. Importantly, although the carboxylic acids required a heteroatom or a carbonyl group in the $\alpha$-position, the mild reaction conditions used tolerate the presence of a wide range of functionalities. Moreover, some carboxylic acids were also successfully used under similar conditions in the decarboxylative homolytic aromatic substitution of 2-(phenylsulfonyl)benzothiazole, to obtain C2substituted benzothiazoles in moderate to good yields. 


\section{Experimental Section}

General Remarks: Reagents and solvents were purchased from different trading houses and were used without further purification, unless otherwise stated. Riboflavin Tetraacetate (RFTA) was prepared from commercial (-)-Riboflavin according to a reported procedure. ${ }^{[23]}$ TLCs were performed on silica gel $60 \mathrm{~F}_{254}$, using aluminium plates and visualized by exposure with UV or stain with PMA $\left(\mathrm{KMnO}_{4}\right.$.for compound 3qa). Flash chromatographies (FC) were carried out on handpacked columns of silica gel 60 (230 - 400 mesh). Infrared (IR) analysis was performed with a JASCO FT/IR 4100 spectrophotometer equipped with an ATR component. LRMS were performed in an AGILENT 6890N mass spectrometer coupled with a gas chromatographer (GC); the mobile phase was helium (2 $\mathrm{mL} / \mathrm{min})$; HP-1 column of $12 \mathrm{~m}$ was used; temperature program starts at $80{ }^{\circ} \mathrm{C}$ for $3 \mathrm{~min}$, then up to $270{ }^{\circ} \mathrm{C}$ with a rate of $15^{\circ} \mathrm{C} / \mathrm{min}$, and $10 \mathrm{~min}$ at $270 \stackrel{\circ}{\circ}$, using the Electron Impact (EI) mode at $70 \mathrm{eV}$ (unless otherwise indicated). HRMS analyses were carried out in an AGILENT 7200 using the Electron Impact (EI) mode at $70 \mathrm{eV}$ by Q-TOF . ${ }^{1} \mathrm{H}-\mathrm{NMR}$ spectra were recorded at 300 or $400 \mathrm{MHz}$ for ${ }^{1} \mathrm{H}-\mathrm{NMR}$ and 75 or $101 \mathrm{MHz}$ for ${ }^{13} \mathrm{C} \mathrm{NMR}$, using $\mathrm{CDCl}_{3}$ as the solvent and referenced to $\mathrm{CDCl}_{3}$ (unless otherwise indicated). ${ }^{13} \mathrm{C}$ NMR spectra were recorded with ${ }^{1} \mathrm{H}$-decoupling at $100 \mathrm{MHz}$ and referenced to $\mathrm{CDCl}_{3}$ at $77.16 \mathrm{ppm}$. Reactions were irradiated in a PhotoRedOx Box Duo (Evoluchem ${ }^{\mathrm{TM}}$ ) equipped with two lamps LED CREE XPE 18W (450-455 nm).

\section{General procedure for the decarboxylative allylation of carboxylic} acids: The corresponding carboxylic acid $(0.25 \mathrm{mmol})$ was added to a 2 dram vial, followed by RFTA (6.8 $\mathrm{mg}, 0.0125 \mathrm{mmol}, 5 \mathrm{~mol}-\%)$, and a solution of 2 ( $0.275 \mathrm{mmol}, 1.1$ equiv) in $\operatorname{MeCN}(2.5 \mathrm{~mL})$. Then, the vial was sealed with a septum and the reaction mixture was degassed by sparging with $\mathrm{Ar}$ for $10 \mathrm{~min}$. Finally, the vial was equipped with an $\mathrm{Ar}$ balloon and the yellow homogeneous solution was stirred at $25^{\circ} \mathrm{C}$ under blue LEDs irradiation $\left(\lambda=455 \mathrm{~nm}, 15 \pm 2 \mathrm{~mW} / \mathrm{cm}^{2}\right)^{[34]}$ until full conversion or no progress of the reaction was observed by TLC or GC (generally $18-22 \mathrm{~h}$ ). The solvent was removed under reduced pressure, affording a residue which was purified by FC.

Butyl 2-methylene-4-phenoxypentanoate (3aa): Compound 3aa was prepared from 2-Phenoxypropanoic acid (1a) following the general procedure. It was purified by FC (100\% Hexane to $90: 10$ Hexane/EtOAc) and obtained as a colorless oil (51 mg, $0.19 \mathrm{mmol}, 77 \%)$ : TLC $R_{f} 0.54$ (95:5 Hexane/EtOAc); IR v 3014, 1712, 1639, 1591, 1489, 1237, 1214, 1157, $746 \mathrm{~cm}^{-1} ;{ }^{1} \mathrm{H}-\mathrm{NMR}\left(300 \mathrm{MHz}, \mathrm{CDCl}_{3}\right) \delta 7.30-7.23(\mathrm{~m}, 2 \mathrm{H}), 6.95-$ $6.89(\mathrm{~m}, 3 \mathrm{H}), 6.24(\mathrm{~d}, J=1.5 \mathrm{~Hz}, 1 \mathrm{H}), 5.67(\mathrm{dd}, J=2.5,1.2 \mathrm{~Hz}, 1 \mathrm{H}), 4.61$ (h, $J=6.2 \mathrm{~Hz}, 1 \mathrm{H}$ ), 4.16 (t, $J=6.6 \mathrm{~Hz}, 2 \mathrm{H}$ ), 2.83 (ddd, $J=13.9,6.6,1.0$ $\mathrm{Hz}, 1 \mathrm{H}), 2.50$ (ddd, $J=13.9,6.3,0.9 \mathrm{~Hz}, 1 \mathrm{H}), 1.71-1.61(\mathrm{~m}, 2 \mathrm{H}), 1.46$ $1.34(\mathrm{~m}, 2 \mathrm{H}), 1.31(\mathrm{~d}, J=6.1 \mathrm{~Hz}, 3 \mathrm{H}), 0.94(\mathrm{t}, J=7.3 \mathrm{~Hz}, 1 \mathrm{H}) \mathrm{ppm} ;{ }^{13} \mathrm{C}$ $\operatorname{NMR}\left(101 \mathrm{MHz}, \mathrm{CDCl}_{3}\right) \delta 167.3(\mathrm{C}), 158.0(\mathrm{C}), 137.2(\mathrm{C}), 129.6(2 \times \mathrm{CH})$, $128.0\left(\mathrm{CH}_{2}\right), 120.7(\mathrm{CH}), 116.0(2 \times \mathrm{CH}), 72.1(\mathrm{CH}), 64.9\left(\mathrm{CH}_{2}\right), 39.3\left(\mathrm{CH}_{2}\right)$ $19.8\left(\mathrm{CH}_{3}\right), 19.4\left(\mathrm{CH}_{2}\right), 13.9\left(\mathrm{CH}_{3}\right) \mathrm{ppm}$; GC $R_{T} 11.15$; LRMS (EI) $\mathrm{m} / \mathrm{z}(\%)$ = $262\left(\mathrm{M}^{+}, 2\right), 169$ (34), 121 (18), 113 (100), 95 (33), 94 (39), 87 (12), 67 (19); HRMS (EI) Calcd. for $\mathrm{C}_{16} \mathrm{H}_{22} \mathrm{O}_{3}-\mathrm{C}_{7} \mathrm{H}_{9} \mathrm{O}$ 153.0916, found 153.0913.

Butyl 2-((2,3-dihydrobenzo[b][1,4]dioxin-2-yl)methyl)acrylate (3ba): Compound 3 ba was prepared from 1,4-Benzodioxane-2-carboxylic acid (1b) following the general procedure. It was purified by FC (100\% Hexane to $95: 5$ Hexane/EtOAc) and obtained as a pale-yellow oil (47 mg, 0.17 mmol, 68\%): TLC $R_{f} 0.57$ (95:5 Hexane/EtOAc); IR v 2980, 2957, 2934 $1716,1381,1274,1244,1210,1193,1156,1146,1105,1091,1060,960$, 869, 755, $696 \mathrm{~cm}^{-1} ;{ }^{1} \mathrm{H}-\mathrm{NMR}\left(300 \mathrm{MHz}, \mathrm{CDCl}_{3}\right) \delta 6.89-6.81(\mathrm{~m}, 4 \mathrm{H}), 6.33$ (d, $J=1.3 \mathrm{~Hz}, 1 \mathrm{H}), 5.73(\mathrm{~d}, J=1.2 \mathrm{~Hz}, 1 \mathrm{H}), 4.41-4.32(\mathrm{~m}, 1 \mathrm{H}), 4.24$ (dd, $J=11.3,2.3 \mathrm{~Hz}, 1 \mathrm{H}), 4.17(\mathrm{t}, J=6.6 \mathrm{~Hz}, 2 \mathrm{H}), 3.93(\mathrm{dd}, J=11.3,6.8 \mathrm{~Hz}$, 1H), 2.72 (ddd, $J=14.3,7.5,0.8 \mathrm{~Hz}, 1 \mathrm{H}$ ), 2.64 (ddd, $J=14.3,5.8,0.9 \mathrm{~Hz}$, $1 \mathrm{H}), 1.72$ - $1.62(\mathrm{~m}, 2 \mathrm{H}), 1.47-1.35(\mathrm{~m}, 2 \mathrm{H}), 0.95(\mathrm{t}, J=7.3 \mathrm{~Hz}, 3 \mathrm{H}) \mathrm{ppm}$;
${ }^{13} \mathrm{C}-\mathrm{NMR}\left(75 \mathrm{MHz}, \mathrm{CDCl}_{3}\right) \delta 166.9(\mathrm{C}), 143.3(\mathrm{C}), 143.1(\mathrm{C}), 135.6(\mathrm{C})$, $128.5\left(\mathrm{CH}_{2}\right), 121.7(\mathrm{CH}), 121.4(\mathrm{CH}), 117.5(\mathrm{CH}), 117.2(\mathrm{CH}), 71.5(\mathrm{CH})$ $67.4\left(\mathrm{CH}_{2}\right), 65.0\left(\mathrm{CH}_{2}\right), 33.8(\mathrm{CH} 2), 30.8\left(\mathrm{CH}_{2}\right), 19.4\left(\mathrm{CH}_{2}\right), 13.6\left(\mathrm{CH}_{3}\right)$ ppm; GC RT $12.44 \mathrm{~min} ; \mathrm{LRMS}(\mathrm{El}) \mathrm{m} / z(\%)=276\left(\mathrm{M}^{+}, 28\right), 135(100), 134$ (10), 121 (12), 111 (16), 110 (11); HRMS (EI) Calcd. for $\mathrm{C}_{16} \mathrm{H}_{20} \mathrm{O}_{4}$ 276.1362 , found 276.1363 .

Butyl 4-(4-chlorophenoxy)-4-methyl-2-methylenepentanoate (3ca): Compound 3ca was prepared from 2-(4-Chlorophenoxy)-2methylpropanoic acid (Clofibric Acid) (1c) $)^{[35]}$ following the general procedure. It was purified by FC (100\% Hexane to 95:5 Hexane/EtOAc) and obtained as a pale-yellow oil ( $47 \mathrm{mg}, 0.15 \mathrm{mmol}, 60 \%)$ : TLC $R_{f} 0.65$ (95:5 Hexane/EtOAc); IR v 2969, 2939, 2878, 1721, 1625, 1584, 1488 , 1482, 1223, 1173, 1152, 1093, 949, 850, 814, $751 \mathrm{~cm}^{-1} ;{ }^{1} \mathrm{H}-\mathrm{NMR}(300 \mathrm{MHz}$, $\left.\mathrm{CDCl}_{3}\right) \delta 7.24-7.17(\mathrm{~m}, 2 \mathrm{H}), 6.93-6.85(\mathrm{~m}, 2 \mathrm{H}), 6.28(\mathrm{~d}, J=1.7 \mathrm{~Hz}, 1 \mathrm{H})$, $5.71-5.66(\mathrm{~m}, 1 \mathrm{H}), 4.15(\mathrm{t}, J=6.6 \mathrm{~Hz}, 2 \mathrm{H}), 2.75(\mathrm{~s}, 2 \mathrm{H}), 1.71-1.57(\mathrm{~m}$, $2 \mathrm{H}), 1.46-1.34(\mathrm{~m}, 2 \mathrm{H}), 1.24(\mathrm{~s}, 6 \mathrm{H}), 6.93(\mathrm{t}, J=7.3 \mathrm{~Hz}, 3) \mathrm{ppm} ;{ }^{13} \mathrm{C}-\mathrm{NMR}$ $\left(101 \mathrm{MHz}, \mathrm{CDCl}_{3}\right) \delta 168.1(\mathrm{C}), 153.9(\mathrm{C}), 137.6(\mathrm{C}), 129.0(2 \times \mathrm{CH}), 128.7$ (C), $128.2\left(\mathrm{CH}_{2}\right), 125.3(2 \times \mathrm{CH}), 80.7(\mathrm{C}), 64.9\left(\mathrm{CH}_{2}\right), 43.4\left(\mathrm{CH}_{2}\right), 30.8$ $\left(\mathrm{CH}_{2}\right), 26.2\left(2 \times \mathrm{CH}_{3}\right), 19.3\left(\mathrm{CH}_{2}\right), 13.8\left(\mathrm{CH}_{3}\right)$ ppm; GC $R_{T} 12.78 \mathrm{~min}$; LRMS (EI) $m / z(\%)=183\left(\mathrm{M}^{+}-\mathrm{C}_{6} \mathrm{H}_{4} \mathrm{ClO}, 13\right), 169(11), 130(29), 128$ (88) 127 (40), 126 (100), 125 (15), 111 (32), 109 (32), 108 (27), 81 (100), 80 (31), 79 (40), 67 (11), 65 (23), 63 (10), 57 (11), 53 (15); HRMS (El) Calcd. for $\mathrm{C}_{17} \mathrm{H}_{23} \mathrm{ClO}_{3} 310.1336$, found 310.1327 .

Butyl 2-methylene-4-( $p$-tolyloxy)butanoate (3da): Compound 3da was prepared from 2-(p-Tolyloxy)acetic acid (1d) following the general procedure. It was purified by FC (100\% Hexane to $95: 5$ Hexane/EtOAc) and obtained as a colorless oil (53 mg, $0.20 \mathrm{mmol}, 80 \%)$ : TLC $R_{f} 0.41$ (95:5 Hexane/EtOAc); IR v 2988, 2956, 2934, 2871, 1740, 1714, 1511, 1372 1235, 1160, 1046, 1045, $817 \mathrm{~cm}^{-1} ;{ }^{1} \mathrm{H}-\mathrm{NMR}\left(300 \mathrm{MHz}, \mathrm{CDCl}_{3}\right) \delta 7.07$ (d, $J=9.2 \mathrm{~Hz}, 2 \mathrm{H}), 6.80(\mathrm{~d}, J=8.6 \mathrm{~Hz}, 2 \mathrm{H}), 6.28(\mathrm{~d}, J=1.3 \mathrm{~Hz}, 1 \mathrm{H}), 5.70$ (dd, $J=2.5,1.2 \mathrm{~Hz}, 1 \mathrm{H}), 4.17(\mathrm{t}, J=6.6 \mathrm{~Hz}, 2 \mathrm{H}), 4.08(\mathrm{t}, J=6.6 \mathrm{~Hz}, 2 \mathrm{H}), 2.78$ $(\mathrm{td}, J=6.6,0.8 \mathrm{~Hz}, 2 \mathrm{H}), 2.28(\mathrm{~s}, 3 \mathrm{H}), 1.71-1.60(\mathrm{~m}, 2 \mathrm{H}), 1.47-1.35(\mathrm{~m}$ $2 \mathrm{H}), 0.94$ (t, $J=7.3 \mathrm{~Hz}, 3 \mathrm{H}) \mathrm{ppm} ;{ }^{13} \mathrm{C}-\mathrm{NMR}\left(101 \mathrm{MHz}, \mathrm{CDCl}_{3}\right) \delta 167.1$ (C), $156.7(\mathrm{C}), 137.1(\mathrm{C}), 130.1(\mathrm{C}), 130.0(2 \times \mathrm{CH}), 127.2\left(\mathrm{CH}_{2}\right), 114.6(2 \times$ $\mathrm{CH}), 66.4\left(\mathrm{CH}_{2}\right), 64.9\left(\mathrm{CH}_{2}\right), 32.1\left(\mathrm{CH}_{2}\right), 30.8\left(\mathrm{CH}_{2}\right), 20.6\left(\mathrm{CH}_{3}\right), 19.4$ $\left(\mathrm{CH}_{2}\right), 13.9\left(\mathrm{CH}_{3}\right) \mathrm{ppm}$; GC $R T 11.82 \mathrm{~min} ; \mathrm{LRMS}(\mathrm{El}) \mathrm{m} / \mathrm{z}(\%)=262\left(\mathrm{M}^{+}\right.$ 2), 155 (17), 108 (21), 107 (13), 99 (100), 91 (14), 81 (11), 77 (10), 53 (11) HRMS (EI) Calcd. for $\mathrm{C}_{16} \mathrm{H}_{22} \mathrm{O}_{3} 262.1569$, found 262.1563 .

Butyl 2-methylene-4-phenoxybutanoate (3ea): Compound 3ea was prepared from 2-Phenoxyacetic acid (1e), following the general procedure but in this case $40 \mathrm{~h}$ of reaction were required. It was purified by $\mathrm{FC}(100 \%$ Hexane to $90: 10$ Hexane/EtOAc) and obtained as a colorless oil (32 mg $0.13 \mathrm{mmol}, 50 \%)$ : TLC $R_{f} 0.65$ (95:5 Hexane/EtOAc); IR $v 2824,1713$ 1598, 1498, 1471, 1294, 1241, 1208, 1160, 1020, 949, 815, 799, 753, 692 $\mathrm{cm}^{-1} ;{ }^{1} \mathrm{H}-\mathrm{NMR}\left(300 \mathrm{MHz}, \mathrm{CDCl}_{3}\right) \delta 7.33-7.23(\mathrm{~m}, 2 \mathrm{H}), 6.99-6.84(\mathrm{~m}, 3 \mathrm{H})$, $6.28(\mathrm{~d}, J=1.2 \mathrm{~Hz}, 1 \mathrm{H}), 5.72(\mathrm{~d}, J=1.2 \mathrm{~Hz}, 1 \mathrm{H}), 4.17(\mathrm{t}, J=6.6 \mathrm{~Hz}, 2 \mathrm{H})$, $4.12(\mathrm{t}, J=6.6 \mathrm{~Hz}, 2 \mathrm{H}), 2.80(\mathrm{td}, J=6.6,0.8 \mathrm{~Hz}, 2 \mathrm{H}), 1.76-1.62(\mathrm{~m}, 2 \mathrm{H})$, 1.41 (dq, $J=14.5,7.3 \mathrm{~Hz}, 2 \mathrm{H}), 0.95(\mathrm{t}, J=7.4 \mathrm{~Hz}, 3 \mathrm{H}) \mathrm{ppm} ;{ }^{13} \mathrm{C}-\mathrm{NMR}(101$ $\left.\mathrm{MHz}, \mathrm{CDCl}_{3}\right) \delta 167.1(\mathrm{C}), 158.8(\mathrm{C}), 137.1(\mathrm{C}), 129.6(2 \times \mathrm{CH}), 127.3\left(\mathrm{CH}_{2}\right)$, $120.9(\mathrm{CH}), 114.7(2 \times \mathrm{CH}), 66.3\left(\mathrm{CH}_{2}\right), 64.9\left(\mathrm{CH}_{2}\right), 32.1\left(\mathrm{CH}_{2}\right), 30.8\left(\mathrm{CH}_{2}\right)$, $19.4\left(\mathrm{CH}_{2}\right), 13.9\left(\mathrm{CH}_{3}\right) \mathrm{ppm}$; GC $R_{T} 13.32 \mathrm{~min} ; \mathrm{LRMS}(\mathrm{EI}) \mathrm{m} / \mathrm{z}(\%)=248$ $\left(\mathrm{M}^{+}, 3\right), 155$ (28), 99 (100), 94 (18), 81 (12), 77 (12); HRMS (EI) Calcd. for $\mathrm{C}_{15} \mathrm{H}_{20} \mathrm{O}_{3} 248.1412$, found 248.1402 .

Butyl 4-(4-chlorophenoxy)-2-methylenebutanoate (3fa): Compound 3fa was prepared from 2-(4-chlorophenoxy)acetic acid (1f) following the general procedure. It was purified by FC (100\% Hexane to $90: 10$ Hexane/EtOAc) and obtained as a colorless oil (28 mg, $0.10 \mathrm{mmol}, 40 \%$ ): TLC $R_{f} 0.60$ (95:5 Hexane/EtOAc); IR $v$ 2958, 2934, 2871, 1713, 1631, 1596, 1579, 1493, 1471, 1281, 1241, 1210, 1159, 1033, $822 \mathrm{~cm}^{-1} ;{ }^{1} \mathrm{H}-\mathrm{NMR}$ $\left(300 \mathrm{MHz}, \mathrm{CDCl}_{3}\right) \delta 7.22(\mathrm{~d}, J=9.0 \mathrm{~Hz}, 2 \mathrm{H}), 6.82(\mathrm{~d}, J=9.0 \mathrm{~Hz}, 2 \mathrm{H}), 6.28$ 
(d, $J=1.2 \mathrm{~Hz}, 1 \mathrm{H}), 5.70(\mathrm{~d}, J=1.2 \mathrm{~Hz}, 1 \mathrm{H}), 4.17(\mathrm{t}, J=6.6 \mathrm{~Hz}, 2 \mathrm{H}), 4.08$ (t, $J=6.6 \mathrm{~Hz}, 2 \mathrm{H}), 2.78(\mathrm{td}, J=6.6,0.8 \mathrm{~Hz}, 2 \mathrm{H}), 1.71-1.62(\mathrm{~m}, 2 \mathrm{H}), 1.41$ (dq, $J=14.4,7.3 \mathrm{~Hz}, 2 \mathrm{H}$ ), 0.94 (t, $J=7.3 \mathrm{~Hz}, 3 \mathrm{H}$ ) ppm; ${ }^{13} \mathrm{C}-\mathrm{NMR}(101$ $\left.\mathrm{MHz}, \mathrm{CDCl}_{3}\right) \delta 167.0(\mathrm{C}), 157.5(\mathrm{C}), 136.9(\mathrm{C}), 129.4(2 \times \mathrm{CH}), 125.7(\mathrm{C})$, $116.0(2 \times \mathrm{CH}), 66.7\left(\mathrm{CH}_{2}\right), 64.9\left(\mathrm{CH}_{2}\right), 32.1\left(\mathrm{CH}_{2}\right), 30.8\left(\mathrm{CH}_{2}\right), 19.4\left(\mathrm{CH}_{2}\right)$, $13.9\left(\mathrm{CH}_{3}\right) \mathrm{ppm}$; GC RT $12.47 \mathrm{~min}$; LRMS (EI) $\mathrm{m} / \mathrm{z}(\%)=282\left(\mathrm{M}^{+}, 3\right), 155$ (21), 128 (15), 99 (100), 81 (10); HRMS (El) Calcd. for $\mathrm{C}_{15} \mathrm{H}_{19} \mathrm{ClO}_{3}$ 282.1023 , found 282.1000 .

Butyl 4-(2-methoxyphenoxy)-2-methylenebutanoate (3ga): Compound 3ga was prepared from 2-(2-methoxyphenoxy)acetic acid (19) following the general procedure. It was purified by FC (100\% Hexane to $90: 10$ Hexane/EtOAc) and obtained as a pale-yellow oil $(38 \mathrm{mg}, 0.14 \mathrm{mmol}$, 55\%): TLC $R_{f} 0.38$ (95:5 Hexane/EtOAc); IR v 2962, 1716, 1631, 1501, $1253,1250,1226,1157,1123,1028,742 \mathrm{~cm}^{-1} ;{ }^{1} \mathrm{H}-\mathrm{NMR}\left(300 \mathrm{MHz}, \mathrm{CDCl}_{3}\right)$ $\delta 6.98-6.85(\mathrm{~m}, 4 \mathrm{H}), 6.29(\mathrm{~d}, J=1.3 \mathrm{~Hz}, 1 \mathrm{H}), 5.74(\mathrm{dd}, J=2.4,1.2 \mathrm{~Hz}$, $1 \mathrm{H}), 4.18(\mathrm{dd}, J=6.8,2.2 \mathrm{~Hz}, 2 \mathrm{H}), 4.16(\mathrm{dd}, J=6.8,1.8 \mathrm{~Hz}, 2 \mathrm{H}), 3.86(\mathrm{~s}$, $3 \mathrm{H}), 2.85(\mathrm{td}, J=7.0,0.9 \mathrm{~Hz}, 2 \mathrm{H}), 1.71-1.61(\mathrm{~m}, 2 \mathrm{H}), 1.41(\mathrm{dq}, J=14.4$, $7.3 \mathrm{~Hz}, 2 \mathrm{H}), 0.94$ (s, 3H) ppm; ${ }^{13} \mathrm{C}-\mathrm{NMR}\left(101 \mathrm{MHz}, \mathrm{CDCl}_{3}\right) \delta 167.1$ (C), $149.7(\mathrm{C}), 148.4(\mathrm{C}), 137.0(\mathrm{C}), 127.5\left(\mathrm{CH}_{2}\right), 121.4(\mathrm{CH}), 121.1(\mathrm{CH})$, $113.7(\mathrm{CH}), 112.2(\mathrm{CH}), 67.7\left(\mathrm{CH}_{2}\right), 64.9\left(\mathrm{CH}_{2}\right), 56.1\left(\mathrm{CH}_{3}\right), 32.1\left(\mathrm{CH}_{2}\right)$, $30.8\left(\mathrm{CH}_{2}\right), 19.4\left(\mathrm{CH}_{2}\right), 13.9\left(\mathrm{CH}_{3}\right) \mathrm{ppm}$; GC $R_{T} 14.34 \mathrm{~min}$; LRMS (EI) $\mathrm{m} / \mathrm{z}$ $(\%)=278\left(\mathrm{M}^{+}, 1 \%\right), 155$ (21), 124 (14), 109 (11), 99 (100), 81 (11); HRMS (EI) Calcd. for $\mathrm{C}_{16} \mathrm{H}_{22} \mathrm{O}_{4} 278.1518$, found 278.1521 .

Butyl 2-((3a $R, 6 R, 6 \mathrm{a} R)-6$-methoxy-2,2-dimethyltetrahydrofuro[3,4d][1,3-dioxol-4-yl)methyl)acrylate (3ha): Compound 3ha was prepared from 2,3-O-isopropylidene-1-O-methyl-D-ribosic acid (1h) following the general procedure. In this case, after $24 \mathrm{~h}$, another $5 \mathrm{~mol}-\%$ of RFTA was added and the reaction was run for another $12 \mathrm{~h}$. It was purified by FC (100\% Hexane to $90: 10$ Hexane/EtOAc) and obtained as a yellow oil (57 $\mathrm{mg}, 0.18 \mathrm{mmol}, 70 \%$, single diastereoisomer): TLC $R_{f} 0.25$ (94:5 Hexane/EtOAc); IR $v$ 2976, 2961, 2939, 1715, 1379, 1266, 1244, 1208, $1187,1154,1144,1105,1090,1058,960,871,695 \mathrm{~cm}^{-1} ;{ }^{1} \mathrm{H}-\mathrm{NMR}(300$ $\left.\mathrm{MHz}, \mathrm{CDCl}_{3}\right) \delta 6.29(\mathrm{~d}, J=1.1 \mathrm{~Hz}, 1 \mathrm{H}), 5.67(\mathrm{q}, J=1.2 \mathrm{~Hz}, 1 \mathrm{H}), 4.95(\mathrm{~s}$, $1 \mathrm{H}), 4.60$ (dd, $J=14.0,6.2 \mathrm{~Hz}, 2 \mathrm{H}), 4.44(\mathrm{td}, J=7.6,0.5 \mathrm{~Hz}, 1 \mathrm{H}), 4.16$ (t, $J=6.6 \mathrm{~Hz}, 2 \mathrm{H}), 3.33(\mathrm{~s}, 3 \mathrm{H}), 2.59(\mathrm{~d}, J=7.7 \mathrm{~Hz}, 2 \mathrm{H}), 1.73-1.60(\mathrm{~m}, 2 \mathrm{H})$, $1.47(\mathrm{~s}, 3 \mathrm{H}), 1.44-1.37(\mathrm{~m}, 2 \mathrm{H}), 1.30(\mathrm{~s}, 3 \mathrm{H}), 0.94(\mathrm{t}, J=7.3 \mathrm{~Hz}, 3 \mathrm{H}) \mathrm{ppm}$; ${ }^{13} \mathrm{C}-\mathrm{NMR}\left(75 \mathrm{MHz}, \mathrm{CDCl}_{3}\right) \delta 166.9(\mathrm{C}), 137.1(\mathrm{C}), 127.4\left(\mathrm{CH}_{2}\right), 112.5(\mathrm{C})$, $109.9(\mathrm{CH}), 85.6(\mathrm{CH}), 85.5(\mathrm{CH}), 83.8(\mathrm{CH}), 64.9\left(\mathrm{CH}_{2}\right), 55.2\left(\mathrm{CH}_{3}\right), 37.4$ $\left(\mathrm{CH}_{2}\right), 30.8\left(\mathrm{CH}_{2}\right), 26.6\left(\mathrm{CH}_{3}\right), 25.2\left(\mathrm{CH}_{3}\right), 19.4\left(\mathrm{CH}_{2}\right), 13.9\left(\mathrm{CH}_{3}\right) \mathrm{ppm} ; \mathrm{GC}$ $R_{T} 11.64 \mathrm{~min} ; \mathrm{LRMS}(\mathrm{EI}) \mathrm{m} / \mathrm{z}(\%)=299\left(\mathrm{M}^{+}-\mathrm{CH}_{3}, 17\right), 211(18), 196(25)$, 174 (10), 173 (100), 171 (10), 169 (33), 141 (11), 140 (16), 139 (12), 123 (12), 122 (14), 119 (26), 117 (29), 116 (10), 115 (29), 97 (18), 95 (34), 94 (11), 87 (19), 85 (23), 59 (28), 58 (11), 57 (17), 55 (16); HRMS (EI) Calcd. for $\mathrm{C}_{16} \mathrm{H}_{26} \mathrm{O}_{6} 314.1729$, found 314.1727 .

Butyl 4-(benzyloxy)-2-methylenepentanoate (3ia): Compound 3ia was prepared from $(R)-(+)-2-($ Benzyloxy)propanoic acid (1i) following the general procedure. It was purified by FC $(100 \%$ Hexane to $95: 5$ Hexane/EtOAc) and obtained as a colorless oil ( $25 \mathrm{mg}, 0.09 \mathrm{mmol}, 35 \%$ ): TLC Rf 0.40 (95:5 Hexane/EtOAc); IR $v$ 2968, 2930, 2901, 1715, 1453, $1376,1125,1073,909,732,697 \mathrm{~cm}^{-1} ;{ }^{1} \mathrm{H}-\mathrm{NMR}\left(300 \mathrm{MHz}, \mathrm{CDCl}_{3}\right) \delta 7.38$ - $7.27(\mathrm{~m}, 5 \mathrm{H}), 6.22(\mathrm{~d}, J=1.7 \mathrm{~Hz}, 1 \mathrm{H}), 5.62(\mathrm{dd}, J=2.6,1.1 \mathrm{~Hz}, 1 \mathrm{H}), 4.53$ (q, $J=11.8 \mathrm{~Hz}, 2 \mathrm{H}$ ), $4.12(\mathrm{t}, J=6.6 \mathrm{~Hz}, 2 \mathrm{H}$ ), 3.73 (sext, $J=6.1 \mathrm{~Hz}, 1 \mathrm{H}$ ), 2.66 (ddd, $J=13.8,6.8,0.9 \mathrm{~Hz}, 1 \mathrm{H}$ ), 2.43 (ddd, $J=13.8,6.0,1.0 \mathrm{~Hz}, 1 \mathrm{H}$ ), $1.68-1.58(\mathrm{~m}, 2 \mathrm{H}), 1.39(\mathrm{dq}, J=14.3,7.3 \mathrm{~Hz}, 2 \mathrm{H}), 1.20(\mathrm{~d}, J=6.1 \mathrm{~Hz}$, $3 \mathrm{H}), 0.94(\mathrm{t}, \mathrm{J}=7.3 \mathrm{~Hz}, 3 \mathrm{H}) \mathrm{ppm} ;{ }^{13} \mathrm{C}-\mathrm{NMR}\left(75 \mathrm{MHz}, \mathrm{CDCl}_{3}\right) \delta 167.4(\mathrm{C})$, $139.0(\mathrm{C}), 137.9(\mathrm{C}), 128.4(2 \times \mathrm{CH}), 127.7(2 \times \mathrm{CH}), 127.5\left(\mathrm{CH}_{2}\right), 127.3$ $(\mathrm{CH}), 73.8(\mathrm{CH}), 70.7\left(\mathrm{CH}_{2}\right), 64.7\left(\mathrm{CH}_{2}\right), 39.6\left(\mathrm{CH}_{2}\right), 30.8\left(\mathrm{CH}_{2}\right), 19.8\left(\mathrm{CH}_{2}\right)$ $19.4\left(\mathrm{CH}_{2}\right), 13.9\left(\mathrm{CH}_{3}\right) \mathrm{ppm}$; GC RT $13.74 \mathrm{~min}$; HRMS (EI) Calcd. for $\mathrm{C}_{17} \mathrm{H}_{24} \mathrm{O}_{3} 276.1725$, found 276.1711 .
Butyl 2-methylene-4-( $p$-tolylthio)butanoate (3ja): Compound 3ja was prepared from 2-( $p$-Tolylthio)acetic acid $(\mathbf{1 j})^{[20]}$ following the general procedure. It was purified by FC (100\% Hexane to $90: 10$ Hexane/EtOAc) and obtained as a colorless oil ( $45 \mathrm{mg}, 0.16 \mathrm{mmol}, 65 \%)$ : TLC $R_{f} 0.65$ (95:5 Hexane/EtOAc); IR v 2962, 2936, 2874, 1710, 1634, 1489, 1177, 1173, $1124,947,803 \mathrm{~cm}^{-1} ;{ }^{1} \mathrm{H}-\mathrm{NMR}\left(300 \mathrm{MHz}, \mathrm{CDCl}_{3}\right) \delta 7.27(\mathrm{~d}, J=8.0 \mathrm{~Hz}, 2 \mathrm{H})$, $7.10(\mathrm{~d}, J=8.0 \mathrm{~Hz}, 2 \mathrm{H}), 6.21(\mathrm{~d}, J=1.2 \mathrm{~Hz}, 1 \mathrm{H}), 5.58(\mathrm{~d}, J=1.2 \mathrm{~Hz}, 1 \mathrm{H})$, $4.14(\mathrm{t}, J=6.6 \mathrm{~Hz}, 2 \mathrm{H}), 3.04(\mathrm{t}, J=7.7 \mathrm{~Hz}, 2 \mathrm{H}), 2.61(\mathrm{t}, J=7.5 \mathrm{~Hz}, 2 \mathrm{H})$, $2.32(\mathrm{~s}, 3 \mathrm{H}), 1.69-1.60(\mathrm{~m}, 2 \mathrm{H}), 1.46-1.33(\mathrm{~m}, 2 \mathrm{H}), 0.94(\mathrm{t}, J=7.3 \mathrm{~Hz}$, $3 \mathrm{H}) \mathrm{ppm} ;{ }^{13} \mathrm{C}-\mathrm{NMR}\left(101 \mathrm{MHz}, \mathrm{CDCl}_{3}\right) \delta 166.9(\mathrm{C}), 138.9(\mathrm{C}), 136.3(\mathrm{C})$, $132.3(\mathrm{C}), 130.2(2 \times \mathrm{CH}), 129.8(2 \times \mathrm{CH}), 126.6\left(\mathrm{CH}_{2}\right), 64.8\left(\mathrm{CH}_{2}\right), 33.2$ $\left(\mathrm{CH}_{2}\right), 32.4\left(\mathrm{CH}_{2}\right), 30.8\left(\mathrm{CH}_{2}\right), 21.1\left(\mathrm{CH}_{3}\right), 19.4\left(\mathrm{CH}_{2}\right), 13.9\left(\mathrm{CH}_{3}\right) \mathrm{ppm} ; \mathrm{GC}$ $R T 12.85 \mathrm{~min}$; LRMS (EI) $\mathrm{m} / \mathrm{z}(\%)=278\left(\mathrm{M}^{+}, 35\right), 205(10), 137(100), 124$ (10), 99 (82), 91 (18); HRMS (EI) Calcd. for $\mathrm{C}_{16} \mathrm{H}_{22} \mathrm{O}_{2} \mathrm{~S} 278.1341$, found 278.1350 .

Butyl 2-methylene-4-oxo-4-phenylbutanoate (3ka): Compound 3ka was prepared from 2-Oxo-2-phenylacetic acid (1k) following the general procedure. It was purified by FC (100\% Hexane to $85: 15$ Hexane/EtOAc) and obtained as a pale-yellow oil ( $30 \mathrm{mg}, 0.12 \mathrm{mmol}, 48 \%$ ): TLC $R_{f} 0.32$ (95:5 Hexane/EtOAc); IR v 2970, 1713, 1685, 1325, 1303, 1212, 1147 , $910,752,730 \mathrm{~cm}^{-1} ;{ }^{1} \mathrm{H}-\mathrm{NMR}\left(300 \mathrm{MHz}, \mathrm{CDCl}_{3}\right) \delta 8.01-7.95(\mathrm{~m}, 2 \mathrm{H}), 7.63$ - $7.53(\mathrm{~m}, 1 \mathrm{H}), 7.51-7.43(\mathrm{~m}, 2 \mathrm{H}), 6.40(\mathrm{~d}, J=1.0 \mathrm{~Hz}, 1 \mathrm{H}), 5.69(\mathrm{~d}, J=$ $1.1 \mathrm{~Hz}, 1 \mathrm{H}), 4.15(\mathrm{t}, J=6.6 \mathrm{~Hz}, 2 \mathrm{H}), 3.99(\mathrm{~d}, J=0.8 \mathrm{~Hz}, 2 \mathrm{H}), 1.68-1.54$ $(\mathrm{m}, 2 \mathrm{H}), 1.35$ (dq, $J=14.4,7.3 \mathrm{~Hz}, 2 \mathrm{H}), 0.89(\mathrm{t}, J=7.3 \mathrm{~Hz}, 3 \mathrm{H}) \mathrm{ppm} ;{ }^{13} \mathrm{C}-$ NMR (101 MHz, CDCl 3 ) $\delta 197.0$ (C), 166.6 (C), 136.7 (C), 135.0 (C), 133.4 $(\mathrm{CH}), 128.8(2 \times \mathrm{CH}), 128.5\left(\mathrm{CH}_{2}\right), 128.4(2 \times \mathrm{CH}), 65.0\left(\mathrm{CH}_{2}\right), 41.8\left(\mathrm{CH}_{2}\right)$, $30.7\left(\mathrm{CH}_{2}\right), 19.3\left(\mathrm{CH}_{2}\right), 13.8\left(\mathrm{CH}_{3}\right) \mathrm{ppm}$; GC RT $13.71 \mathrm{~min}$; LRMS (EI) $\mathrm{m} / \mathrm{z}$ $(\%)=173\left(\mathrm{M}^{+}-\mathrm{C}_{4} \mathrm{H}_{9} \mathrm{O}, 7\right), 172(12), 106$ (8), 105 (100), 77 (28); HRMS (EI) Calcd. for $\mathrm{C}_{15} \mathrm{H}_{18} \mathrm{O}_{3} 246.1256$, found 246.1245 .

Butyl 2-methylene-4-(phenylamino)butanoate (3la): Compound 3la was prepared from $(N)$-Phenylglycine (11) following the general procedure. It was purified by FC (100\% Hexane to $90: 10 \mathrm{Hexane} / \mathrm{EtOAC})$ and obtained as a colorless oil (39 mg, $0.15 \mathrm{mmol}, 60 \%)$ : TLC Rf 0.29 (95:5 Hexane/EtOAc); IR v 2969, 2901, 1707, 1603, 1508, 1215, 1066, 908, 754, $731 \mathrm{~cm}^{-1}$; ${ }^{1} \mathrm{H}-\mathrm{NMR}\left(300 \mathrm{MHz}, \mathrm{CDCl}_{3}\right) \delta 7.23-7.14(\mathrm{~m}, 2 \mathrm{H}), 6.73$ (t, $J=7.3$ $\mathrm{Hz}, 1 \mathrm{H}), 6.67(\mathrm{~d}, J=7.7 \mathrm{~Hz}, 2 \mathrm{H}), 6.25(\mathrm{~d}, J=1.3 \mathrm{~Hz}, 1 \mathrm{H}), 5.63(\mathrm{~d}, J=1.2$ $\mathrm{Hz}, 1 \mathrm{H}), 4.17(\mathrm{t}, J=6.6 \mathrm{~Hz}, 2 \mathrm{H}), 3.31(\mathrm{t}, J=6.8 \mathrm{~Hz}, 2 \mathrm{H}), 2.64(\mathrm{td}, J=6.8$, $0.7 \mathrm{~Hz}, 2 \mathrm{H}), 1.77-1.59(\mathrm{~m}, 2 \mathrm{H}), 1.43-1.40(\mathrm{~m}, 2 \mathrm{H}), 0.95(\mathrm{t}, J=7.3 \mathrm{~Hz}$, 3H) ppm; ${ }^{13} \mathrm{C}-\mathrm{NMR}\left(101 \mathrm{MHz}, \mathrm{CDCl}_{3}\right) \delta 167.2(\mathrm{C}), 147.5$ (C), 138.3 (C), $129.4(2 \times \mathrm{CH}), 126.9\left(\mathrm{CH}_{2}\right), 118.0(\mathrm{CH}), 113.5(2 \times \mathrm{CH}), 65.0\left(\mathrm{CH}_{2}\right), 43.4$ $\left(\mathrm{CH}_{2}\right), 32.0\left(\mathrm{CH}_{2}\right), 30.8\left(\mathrm{CH}_{2}\right), 19.4\left(\mathrm{CH}_{2}\right), 13.9\left(\mathrm{CH}_{3}\right) \mathrm{ppm}$; GC $R_{T} 11.99$ $\mathrm{min} ;$ LRMS (EI) $\mathrm{m} / \mathrm{z}(\%)=247\left(\mathrm{M}^{+}, 14\right), 173(24), 106(100), 105(15), 104$ (11), 77 (21); HRMS (EI) Calcd. for $\mathrm{C}_{15} \mathrm{H}_{21} \mathrm{NO}_{2} 247.1572$, found 247.1565.

Butyl 2-methylene-4-(phenylamino)pentanoate (3ma): Compound 3ma was prepared from $(M)$-Phenylalanine $(\mathbf{1} \mathbf{m})^{[36]}$ following the general procedure. It was purified by FC (100\% Hexane to 95:5 Hexane/EtOAc) and obtained as a pale-yellow oil ( $26 \mathrm{mg}, 0.10 \mathrm{mmol}, 40 \%$ ): TLC $R_{f} 0.46$ (95:5 Hexane/EtOAc); IR v 3398, 2961, 2929, 2870, 1710, 1602, 1500, $1317,1255,1215,1161,946,744,689 \mathrm{~cm}^{-1} ;{ }^{1} \mathrm{H}-\mathrm{NMR}\left(300 \mathrm{MHz}, \mathrm{CDCl}_{3}\right)$ $\delta 7.19-7.12(\mathrm{~m}, 2 \mathrm{H}), 6.69-6.60(\mathrm{~m}, 3 \mathrm{H}), 6.22(\mathrm{~d}, J=1.5 \mathrm{~Hz}, 1 \mathrm{H}), 5.59$ (d, $J=1.3 \mathrm{~Hz}, 1 \mathrm{H}), 4.17(\mathrm{t}, J=6.7 \mathrm{~Hz}, 2 \mathrm{H}), 3.71(\mathrm{~h}, J=6.4 \mathrm{~Hz}, 1 \mathrm{H}), 2.73$ (ddd, $J=13.8,6.4,1.0 \mathrm{~Hz}, 1 \mathrm{H}$ ), 2.32 (ddd, $J=13.8,6.9,0.8 \mathrm{~Hz}, 1 \mathrm{H}$ ), 1.71 - $1.62(\mathrm{~m}, 2 \mathrm{H}), 1.47-1.34(\mathrm{~m}, 2 \mathrm{H}), 1.18(\mathrm{~d}, J=6.4 \mathrm{~Hz}, 3 \mathrm{H}), 0.94(\mathrm{t}, J=$ 7.3Hz, 3H) ppm; ${ }^{13} \mathrm{C}-\mathrm{NMR}\left(101 \mathrm{MHz}, \mathrm{CDCl}_{3}\right) \delta 167.6$ (C), 147.4 (C), 138.3 (C), $129.4(2 \times \mathrm{CH}), 127.3\left(\mathrm{CH}_{2}\right), 117.2(\mathrm{CH}), 113.3(2 \times \mathrm{CH}), 64.9\left(\mathrm{CH}_{2}\right)$, $48.1(\mathrm{CH}), 39.4\left(\mathrm{CH}_{2}\right), 30.9\left(\mathrm{CH}_{2}\right), 20.7\left(\mathrm{CH}_{3}\right), 19.4\left(\mathrm{CH}_{2}\right), 13.9\left(\mathrm{CH}_{3}\right) \mathrm{ppm}$; GC $R_{T}=13.98 \mathrm{~min}$; LRMS (EI) $m / z(\%)=261\left(\mathrm{M}^{+}, 4\right), 121(11), 120(100)$, 77 (11); HRMS (EI) Calcd. for $\mathrm{C}_{16} \mathrm{H}_{23} \mathrm{NO}_{2} 261.1729$, found 261.1735.

Butyl 4-((2-methoxyphenyl)amino)-2-methylenebutanoate (3na): Compound 3na was prepared from (2-Methoxyphenyl)glycine $(\mathbf{1 n})^{[22]}$ 
following the general procedure. It was purified by FC $(100 \%$ Hexane to 95:5 Hexane/EtOAc) and obtained as a pale-yellow oil ( $31 \mathrm{mg}, 0.11 \mathrm{mmol}$, 45\%): TLC $R_{f} 0.45$ (95:5 Hexane/EtOAc); IR v 2960, 2901, 1713, 1602 $1512,1244,1222,1156,1124,1028,946 \mathrm{~cm}^{-1} ;{ }^{1} \mathrm{H}-\mathrm{NMR}\left(300 \mathrm{MHz}, \mathrm{CDCl}_{3}\right)$ $\delta 6.93-6.83(\mathrm{~m}, 1 \mathrm{H}), 6.81-6.74(\mathrm{~m}, 1 \mathrm{H}), 6.74-6.64(\mathrm{~m}, 2 \mathrm{H}), 6.25(\mathrm{~d}, J$ $=1.3 \mathrm{~Hz}, 1 \mathrm{H}), 5.62(\mathrm{~d}, J=1.2 \mathrm{~Hz}, 1 \mathrm{H}), 4.17(\mathrm{t}, J=6.6 \mathrm{~Hz}, 2 \mathrm{H}), 3.84(\mathrm{~s}$, $3 \mathrm{H}), 3.32(\mathrm{t}, J=7.0 \mathrm{~Hz}, 2 \mathrm{H}), 2.66(\mathrm{t}, J=6.7 \mathrm{~Hz}, 2 \mathrm{H}), 1.75-1.60(\mathrm{~m}, 2 \mathrm{H})$, $1.42(\mathrm{dq}, J=14.4,7.3 \mathrm{~Hz}, 2 \mathrm{H}), 0.95(\mathrm{t}, J=7.3 \mathrm{~Hz}, 3 \mathrm{H}) \mathrm{ppm} ;{ }^{1{ }^{3} \mathrm{C}-N M R}(101$ $\left.\mathrm{MHz}, \mathrm{CDCl}_{3}\right) \delta 167.2(\mathrm{C}), 147.2(\mathrm{C}), 138.3\left(\mathrm{CH}_{2}\right), 137.6(\mathrm{C}), 126.7(\mathrm{CH})$, $121.5(\mathrm{CH}), 117.1(\mathrm{C}), 110.5(\mathrm{CH}), 109.7(\mathrm{CH}), 64.9\left(\mathrm{CH}_{2}\right), 55.6\left(\mathrm{CH}_{3}\right)$, $43.0\left(\mathrm{CH}_{2}\right), 32.0\left(\mathrm{CH}_{2}\right), 30.8\left(\mathrm{CH}_{2}\right), 19.4\left(\mathrm{CH}_{2}\right), 13.9\left(\mathrm{CH}_{3}\right) \mathrm{ppm}$; GC $R T$ $15.0 \mathrm{~min}$; LRMS (EI) $\mathrm{m} / \mathrm{z}(\%)=277\left(\mathrm{M}^{+}, 16\right), 136(100), 121(14), 120(17)$; HRMS (EI) Calcd. for $\mathrm{C}_{16} \mathrm{H}_{23} \mathrm{NO}_{3} 277.1678$, found 277.1678.

\section{Butyl 4-((4-bromophenyl)amino)-2-methylenebutanoate (30a)} Compound 3oa was prepared from (4-Bromophenyl)glycine (10) ${ }^{[22]}$ following the general procedure. It was purified by FC $(100 \%$ Hexane to $90: 10 \mathrm{Hexane} / \mathrm{EtOAc}$ ) and obtained as a pale-yellow oil ( $30 \mathrm{mg}, 0.09 \mathrm{mmol}$ $35 \%)$ : TLC $R_{f} 0.44$ (95:5 Hexane/EtOAc); ${ }^{1} \mathrm{H}-\mathrm{NMR}\left(300 \mathrm{MHz}, \mathrm{CDCl}_{3}\right) \delta$ 7.27 - $7.21(\mathrm{~m}, 2 \mathrm{H}), 6.53-6.48(\mathrm{~m}, 2 \mathrm{H}), 6.24(\mathrm{~d}, J=1.3 \mathrm{~Hz}, 1 \mathrm{H}), 5.62(\mathrm{~d}$, $J=1.2 \mathrm{~Hz}, 1 \mathrm{H}$ ), $4.17(\mathrm{t}, J=6.6 \mathrm{~Hz}, 2 \mathrm{H}), 3.26(\mathrm{t}, J=6.8 \mathrm{~Hz}, 2 \mathrm{H}$ ), 2.61 (td, $J=6.8,0.7 \mathrm{~Hz}, 2 \mathrm{H}), 1.71-1.62(\mathrm{~m}, 2 \mathrm{H}), 1.47-1.35(\mathrm{~m}, 2 \mathrm{H}), 0.95(\mathrm{t}, J=$ $7.3 \mathrm{~Hz}, 3 \mathrm{H}) \mathrm{ppm} ;{ }^{13} \mathrm{C}-\mathrm{NMR}\left(101 \mathrm{MHz}, \mathrm{CDCl}_{3}\right) \delta 167.2(\mathrm{C}), 146.9(\mathrm{C}), 138.2$ (C), $132.1(2 \times \mathrm{CH}), 127.0(\mathrm{CH}), 114.7(2 \times \mathrm{CH}), 109.2(\mathrm{C}), 65.0\left(\mathrm{CH}_{2}\right)$, 43.2 $\left(\mathrm{CH}_{2}\right), 31.9\left(\mathrm{CH}_{2}\right), 30.8\left(\mathrm{CH}_{2}\right), 19.4\left(\mathrm{CH}_{2}\right), 13.9\left(\mathrm{CH}_{3}\right)$ ppm; GC $R_{T}$ $14.10 \mathrm{~min}$; LRMS (EI) $\mathrm{m} / \mathrm{z}(\%)=327\left(\mathrm{M}^{+}{ }^{+11} \mathrm{Br}, 13\right), 325\left(\mathrm{M}^{+}{ }^{79} \mathrm{Br}, 14\right), 186$ (95), 185 (11), 184 (100), 105 (11); HRMS (EI) Calcd. for $\mathrm{C}_{15} \mathrm{H}_{20} \mathrm{BrNO}_{2}$ 325.0677 , found 325.0650 .

Butyl 2-methylene-4-(1H-pyrrol)-1-yl)pentanoate (3pa): Compound 3pa was prepared from 2-(1H-Pyrrol-1-yl)propanoic acid (1p) $)^{[37]}$ following the general procedure. It was purified by FC $(100 \%$ Hexane to $95: 5$ Hexane/EtOAc) and obtained as a colorless oil ( $35 \mathrm{mg}, 0.15 \mathrm{mmol}, 58 \%$ ): TLC Rf 0.25 (95:5 Hexane/EtOAc); IR $v$ 3023, 2955, 2928, 2871, 1707, 1631, 1487, 1220, 1165, $749 \mathrm{~cm}^{-1} ;{ }^{1} \mathrm{H}-\mathrm{NMR}\left(300 \mathrm{MHz}, \mathrm{CDCl}_{3}\right) \delta 6.67$ (t, $J$ $=2.1 \mathrm{~Hz}, 2 \mathrm{H}), 6.11(\mathrm{t}, J=2.0 \mathrm{~Hz}, 2 \mathrm{H}), 6.08(\mathrm{~d}, J=1.4 \mathrm{~Hz}, 1 \mathrm{H}), 5.31(\mathrm{~d}, J$ $=1.2 \mathrm{~Hz}, 1 \mathrm{H}), 4.32(\mathrm{dq}, J=13.4,6.8 \mathrm{~Hz}, 1 \mathrm{H}), 4.16(\mathrm{t}, J=6.6 \mathrm{~Hz}, 2 \mathrm{H}), 2.75$ - $2.59(\mathrm{~m}, 2 \mathrm{H}), 1.73-1.49(\mathrm{~m}, 2 \mathrm{H}), 1.47(\mathrm{t}, J=5.3 \mathrm{~Hz}, 2 \mathrm{H}), 1.45-1.35(\mathrm{~m}$, $2 \mathrm{H}), 0.96(\mathrm{t}, J=7.3 \mathrm{~Hz}, 3 \mathrm{H}) \mathrm{ppm} ;{ }^{13} \mathrm{C}-\mathrm{NMR}\left(75 \mathrm{MHz}, \mathrm{CDCl}_{3}\right) \delta 170.0(\mathrm{C})$, $136.9(\mathrm{C}), 127.8\left(\mathrm{CH}_{2}\right), 118.6(2 \times \mathrm{CH}), 107.8(2 \times \mathrm{CH}), 64.9\left(\mathrm{CH}_{2}\right), 54.4$ $(\mathrm{CH}), 41.5\left(\mathrm{CH}_{2}\right), 30.8\left(\mathrm{CH}_{2}\right), 21.7\left(\mathrm{CH}_{3}\right), 19.4\left(\mathrm{CH}_{2}\right), 13.9\left(\mathrm{CH}_{3}\right) \mathrm{ppm} ; \mathrm{GC}$ $R_{T} 12.12 \mathrm{~min}$; LRMS (EI) $\mathrm{m} / \mathrm{z}(\%)=235\left(\mathrm{M}^{+}, 28\right), 178(53), 163(12), 134$ (77), 133 (17), 132 (19), 118 (17), 117 (15), 94 (100); HRMS (EI) Calcd. for $\mathrm{C}_{14} \mathrm{H}_{21} \mathrm{NO}_{2} 235.1572$, found 235.1568 .

Butyl 2-(((3r,5r,7r)-adamantan-1-yl)methyl)acrylate (3qa): It was prepared from adamantane-1-carboxylic acid (1q, 2 equiv) following the general procedure, but degasification was performed by three cycles of freeze-pump-thaw. It was purified by FC (100\% Hexane to $95: 5$ Hexane/EtOAc) and obtained as a colorless oil ( $27 \mathrm{mg}, 0.10 \mathrm{mmol}, 40 \%$ ): TLC $R_{f} 0.88$ (90:10 Hexane/EtOAc); IR v 2903, 2847, 1718, 1176, 1132 , 1059, $806 \mathrm{~cm}^{-1} ;{ }^{1} \mathrm{H}-\mathrm{NMR}\left(300 \mathrm{MHz}, \mathrm{CDCl}_{3}\right) 6.19(\mathrm{~d}, J=1.9 \mathrm{~Hz}, 1 \mathrm{H}), 5.44$ $-5.40(\mathrm{~m}, 1 \mathrm{H}), 4.15(\mathrm{t}, J=6.7 \mathrm{~Hz}, 2 \mathrm{H}), 2.17(\mathrm{~s}, 2 \mathrm{H}), 1.95(\mathrm{br} \mathrm{s}, 3 \mathrm{H}), 1.74$ $-1.57(\mathrm{~m}, 12 \mathrm{H}), 1.47-1.46(\mathrm{~m}, 4 \mathrm{H}), 0.97(\mathrm{t}, J=7.3 \mathrm{~Hz}, 3 \mathrm{H}) \mathrm{ppm} ;{ }^{13} \mathrm{C}$ NMR $\left(101 \mathrm{MHz}, \mathrm{CDCl}_{3}\right) \delta 168.5(\mathrm{C}), 137.8(\mathrm{C}), 127.0\left(\mathrm{CH}_{2}\right), 64.67\left(\mathrm{CH}_{2}\right), 45.6$ $\left(\mathrm{CH}_{2}\right), 42.2\left(3 \times \mathrm{CH}_{2}\right), 37.1\left(3 \times \mathrm{CH}_{2}\right), 33.4(\mathrm{C}), 30.8\left(\mathrm{CH}_{2}\right), 28.8(3 \times \mathrm{CH})$, $19.39\left(\mathrm{CH}_{2}\right), 13.88\left(\mathrm{CH}_{3}\right) \mathrm{ppm}$; GC RT $12.33 \mathrm{~min}$; LRMS (EI) $\mathrm{m} / \mathrm{z}(\%)=$ $276\left(\mathrm{M}^{+}, 1\right), 203(2), 136$ (11), 135 (100), 79 (10); HRMS (EI) Calcd. for $\mathrm{C}_{18} \mathrm{H}_{28} \mathrm{O}_{2} 276.2089$, found 276.2088 .

Ethyl 2-methylene-4-phenoxypentanoate (3ab): Compound 3ab was prepared from $\mathbf{2 b}$ after $12 \mathrm{~h}$, following the general procedure. It was purified by FC (100\% Hexane to $90: 10$ Hexane/EtOAc) and obtained as a colorless oil (45 mg, $0.19 \mathrm{mmol}, 76 \%$ ): TLC $R f 0.54$ (98:2 Hexane/EtOAc);
IR $v 2985,2976,2901,1712,1599,1586,1491,1406,1394,1381,1241$ $1231,1174,1157,1076,1066,947,891,752,692 \mathrm{~cm}^{-1} ;{ }^{1} \mathrm{H}-\mathrm{NMR}(300 \mathrm{MHz}$, $\left.\mathrm{CDCl}_{3}\right) \delta 7.31-7.22(\mathrm{~m}, 2 \mathrm{H}), 6.95-6.92(\mathrm{~m}, 3 \mathrm{H}), 6.25(\mathrm{~d}, J=1.5 \mathrm{~Hz}, 1 \mathrm{H})$, $5.68(\mathrm{~d}, J=1.2 \mathrm{~Hz}, 1 \mathrm{H}), 4.61(\mathrm{~h}, J=6.2 \mathrm{~Hz}, 1 \mathrm{H}), 4.22(\mathrm{q}, J=7.1 \mathrm{~Hz}, 2 \mathrm{H})$, 2.83 (ddd, $J=13.9,6.5,0.9 \mathrm{~Hz}, 1 \mathrm{H}$ ), 2.50 (ddd, $J=13.9,6.4,0.8 \mathrm{~Hz}, 1 \mathrm{H}$ ), 1.31 (d, $J=6.2 \mathrm{~Hz}, 3 \mathrm{H}), 1.30$ (t, $J=7.2 \mathrm{~Hz}, 3 \mathrm{H}) \mathrm{ppm} ;{ }^{13} \mathrm{C}-\mathrm{NMR}(101 \mathrm{MHz}$, $\left.\mathrm{CDCl}_{3}\right) \delta 167.2(\mathrm{C}), 158.0(\mathrm{C}), 137.1(\mathrm{C}), 129.6(2 \times \mathrm{CH}), 128.0\left(\mathrm{CH}_{2}\right)$, $120.7(\mathrm{CH}), 115.9(2 \times \mathrm{CH}), 72.1(\mathrm{CH}), 61.0\left(\mathrm{CH}_{2}\right), 39.2\left(\mathrm{CH}_{2}\right), 19.8\left(\mathrm{CH}_{3}\right)$, $14.3\left(\mathrm{CH}_{3}\right) \mathrm{ppm}$; GC RT $10.05 \mathrm{~min}$; LRMS (EI) $\mathrm{m} / \mathrm{z}(\%)=234\left(\mathrm{M}^{+}, 4\right), 189$ (11), 141 (100), 121 (25), 113 (73), 95 (57), 94 (73), 87 (27), 68 (11), 67 (41), 66 (11), 65 (13); HRMS (EI) Calcd. for $\mathrm{C}_{14} \mathrm{H}_{8} \mathrm{O}_{3} 234.1256$, found 234.162 .

Benzyl 2-methylene-4-phenoxypentanoate (3ac): Compound 3ac was prepared from $2 \mathrm{c}$ after $12 \mathrm{~h}$, following the general procedure. It was purified by FC (100\% Hexane to $90: 10$ Hexane/EtOAc) and obtained as a pale-yellow oil (47 mg, $0.16 \mathrm{mmol}, 65 \%)$ : TLC $R_{f} 0.47 \quad(95: 5$ Hexane/EtOAc); IR $v$ 3014, 1712, 1639, 1591, 1489, 1237, 1214, 1157, $746 \mathrm{~cm}^{-1} ;{ }^{1} \mathrm{H}-\mathrm{NMR}\left(400 \mathrm{MHz}, \mathrm{CDCl}_{3}\right) \delta 7.40-7.32(\mathrm{~m}, 5 \mathrm{H}), 7.25-7.19(\mathrm{~m}$ $2 \mathrm{H}), 6.95-6.84(\mathrm{~m}, 3 \mathrm{H}), 6.31(\mathrm{~d}, J=1.4 \mathrm{~Hz}, 1 \mathrm{H}), 5.72(\mathrm{~d}, J=1.2 \mathrm{~Hz}, 1 \mathrm{H})$, $5.21(\mathrm{~s}, 2 \mathrm{H}), 4.61(\mathrm{~h}, J=6.2 \mathrm{~Hz}, 1 \mathrm{H}), 2.85$ (ddd, $J=13.9,6.7,0.9 \mathrm{~Hz}, 1 \mathrm{H}$ ) 2.53 (ddd, $J=13.9,6.2,0.8 \mathrm{~Hz}, 1 \mathrm{H}), 1.30(\mathrm{~d}, J=6.1 \mathrm{~Hz}, 3 \mathrm{H}) \mathrm{ppm} ;{ }^{13} \mathrm{C}-$ $\operatorname{NMR}\left(101 \mathrm{MHz}, \mathrm{CDCl}_{3}\right) \delta 167.0$ (C), 158.0 (C), 136.9 (C), 136.0 (C), 129.6 $(2 \times \mathrm{CH}), 128.73(2 \times \mathrm{CH}), 128.65(\mathrm{CH}), 128.4(2 \times \mathrm{CH}), 128.3\left(\mathrm{CH}_{2}\right)$, $120.7(\mathrm{CH}), 115.9(2 \times \mathrm{CH}), 72.0(\mathrm{CH}), 66.8\left(\mathrm{CH}_{2}\right), 39.3\left(\mathrm{CH}_{2}\right), 19.8\left(\mathrm{CH}_{3}\right)$ ppm; GC $R_{T} 13.28 \mathrm{~min}$; LRMS (EI) $\mathrm{m} / \mathrm{z}(\%)=203\left(\mathrm{M}^{+}-\mathrm{C}_{6} \mathrm{H}_{5} \mathrm{O}, 19\right), 94(11)$ 91 (100); HRMS (EI) Calcd. for $\mathrm{C}_{19} \mathrm{H}_{20} \mathrm{O}_{3}$ 296.1412, found 296.1406.

Allyl 2-methylene-4-phenoxypentanoate (3ad): Compound 3ad was prepared from $\mathbf{2 d}$ after $12 \mathrm{~h}$, following the general procedure. It was purified by FC $(100 \%$ Hexane to $90: 10$ Hexane/EtOAc) and obtained as a colorless oil ( $39 \mathrm{mg}, 0.16 \mathrm{mmol}, 65 \%)$ : TLC $R_{f} 0.49$ (95:5 Hexane/EtOAc); IR $v 1720,1636,1493,1211,1169,908,748 \mathrm{~cm}^{-1} ;{ }^{1} \mathrm{H}-\mathrm{NMR}(300 \mathrm{MHz}$, $\left.\mathrm{CDCl}_{3}\right) \delta 7.30-7.24(\mathrm{~m}, 2 \mathrm{H}), 6.95-6.89(\mathrm{~m}, 3 \mathrm{H}), 6.29(\mathrm{~d}, J=1.4 \mathrm{~Hz}, 1 \mathrm{H})$ 5.95 (ddt, $J=17.2,10.5,5.7 \mathrm{~Hz}, 1 \mathrm{H}), 5.71(\mathrm{~d}, J=1.2 \mathrm{~Hz}, 1 \mathrm{H}), 5.34$ (dq, $J$ $=17.2,1.5 \mathrm{~Hz}, 1 \mathrm{H}$ ), 5.25 (ddd, $J=10.4,2.6,1.3 \mathrm{~Hz}, 1 \mathrm{H}$ ), 4.67 (dt, $J=5.7$ $1.4 \mathrm{~Hz}, 2 \mathrm{H}), 4.64-4.59(\mathrm{~m}, 1 \mathrm{H}), 2.84$ (ddd, $J=13.9,6.6,0.9 \mathrm{~Hz}, 1 \mathrm{H}), 2.52$ (ddd, $J=13.9,6.2,0.9 \mathrm{~Hz}, 1 \mathrm{H}$ ), $1.31(\mathrm{~d}, J=6.1 \mathrm{~Hz}, 3 \mathrm{H}) \mathrm{ppm} ;{ }^{13} \mathrm{C}-\mathrm{NMR}$ $\left(101 \mathrm{MHz}, \mathrm{CDCl}_{3}\right) \delta 166.8(\mathrm{C}), 158.0$ (C), $136.9(\mathrm{C}), 132.2(\mathrm{CH}), 129.6(2$ $\times \mathrm{CH}), 128.5\left(\mathrm{CH}_{2}\right), 120.8(\mathrm{CH}), 118.4\left(\mathrm{CH}_{2}\right), 115.9(2 \times \mathrm{CH}), 72.0(\mathrm{CH})$, $65.6\left(\mathrm{CH}_{2}\right), 39.2\left(\mathrm{CH}_{2}\right), 19.8\left(\mathrm{CH}_{3}\right) \mathrm{ppm}$; GC RT $10.45 \mathrm{~min}$; LRMS (EI) $\mathrm{m} / \mathrm{z}$ $(\%)=246\left(\mathrm{M}^{+}, 5\right), 154(10), 153(100), 121(25), 107(15), 95$ (25), $94(54)$, 91(10), 67 (30), 65 (10); HRMS (EI) Calcd. for $\mathrm{C}_{15} \mathrm{H}_{18} \mathrm{O}_{3}-\mathrm{C}_{6} \mathrm{H}_{5} \mathrm{O}$ 153.0916, found 153.0916 .

((4-chloropent-4-en-2-yl)oxy)benzene (3ae): Compound 3ae was prepared from $2 \mathrm{e}$ after $12 \mathrm{~h}$, following the general procedure. It was purified by FC $(100 \%$ Hexane to $95: 5$ Hexane/EtOAc) and obtained as a colorless oil ( $30 \mathrm{mg}, 0.15 \mathrm{mmol}, 60 \%$ ): TLC $R f 0.67$ (98:2 Hexane/EtOAc); IR $v 3025,2943,1718,1635,1597,1587,1494,1380,1240,1220,1081$, 1016, $754 \mathrm{~cm}^{-1} ;{ }^{1} \mathrm{H}-\mathrm{NMR}\left(300 \mathrm{MHz}, \mathrm{CDCl}_{3}\right) \delta 7.32-7.25(\mathrm{~m}, 2 \mathrm{H}), 6.97-$ $6.90(\mathrm{~m}, 3 \mathrm{H}), 5.25(\mathrm{~s}, 2 \mathrm{H}), 4.70(\mathrm{~h}, J=6.2 \mathrm{~Hz}, 1 \mathrm{H}), 2.85$ (dd, $J=14.5,6.3$ $\mathrm{Hz}, 1 \mathrm{H}), 2.50$ (dd, $J=14.4,6.5 \mathrm{~Hz}, 1 \mathrm{H}), 1.35(\mathrm{~d}, J=6.1 \mathrm{~Hz}, 3 \mathrm{H}) \mathrm{ppm} ;{ }^{13} \mathrm{C}-$ $\operatorname{NMR}\left(75 \mathrm{MHz}, \mathrm{CDCl}_{3}\right) \delta 157.8(\mathrm{C}), 138.9(\mathrm{C}), 129.7(2 \times \mathrm{CH}), 121.1(\mathrm{CH})$, $116.2(2 \times \mathrm{CH}), 115.0\left(\mathrm{CH}_{2}\right), 71.2\left(\mathrm{CH}_{2}\right), 46.1(\mathrm{CH}), 19.5\left(\mathrm{CH}_{3}\right) \mathrm{ppm} ; \mathrm{GC}$ $R_{T} 8.18 \mathrm{~min}$; LRMS (EI) $\mathrm{m} / \mathrm{z}(\%)=196\left(\mathrm{M}^{+}, 11\right), 121(41), 94(100), 77(16)$; HRMS (EI) Calcd. for $\mathrm{C}_{11} \mathrm{H}_{13} \mathrm{ClO} 196.0655$, found 196.0658 .

((4-phenoxypent-1-en-2-yl)sulfonyl)benzene (3af): Compound 3af was prepared from $\mathbf{2 f}$, following the general procedure. In this case the reaction was run during $40 \mathrm{~h}$ employing 2 equiv of $1 \mathrm{a}$ and 1 equiv of $\mathbf{2 f}$. It was purified by FC $(100 \%$ Hexane to $75: 25$ Hexane/EtOAc) and obtained as a white solid (31 mg, $0.10 \mathrm{mmol}, 68 \%$ ): TLC Rf 0.11 (95:5 Hexane/EtOAc); IR $v$ 3063, 2980, 2930, 1598, 1586, 1491, 1446, 1304, 1292, 1237, 1173 , 
1139, 1081, 952, $689 \mathrm{~cm}^{-1} ;{ }^{1} \mathrm{H}-\mathrm{NMR}\left(300 \mathrm{MHz}, \mathrm{CDCl}_{3}\right) \delta 7.92-7.82(\mathrm{~m}$, $2 \mathrm{H}), 7.65-7.57(\mathrm{~m}, 1 \mathrm{H}), 7.55-7.45(\mathrm{~m}, 2 \mathrm{H}), 7.30-7.20(\mathrm{~m}, 2 \mathrm{H}), 6.98-$ $6.88(\mathrm{~m}, 1 \mathrm{H}), 6.84-6.76(\mathrm{~m}, 2 \mathrm{H}), 6.44(\mathrm{br} \mathrm{s}, 1 \mathrm{H}), 5.93(\mathrm{br} \mathrm{s}, 1 \mathrm{H}), 4.60-$ $4.50(\mathrm{~m}, 1 \mathrm{H}), 2.66$ (ddd, $J=15.4,7.4,0.8 \mathrm{~Hz}, 1 \mathrm{H}$ ), 2.49 (ddd, $J=15.4,5.2$, $1.0 \mathrm{~Hz}, 1 \mathrm{H}), 1.26(\mathrm{~d}, J=6.1 \mathrm{~Hz}, 3 \mathrm{H}) \mathrm{ppm} ;{ }^{13} \mathrm{C}-\mathrm{NMR}\left(101 \mathrm{MHz}, \mathrm{CDCl}_{3}\right) \delta$ 157.4 (C), $146.7(\mathrm{C}), 138.7(\mathrm{C}), 133.7(\mathrm{CH}), 129.7(2 \times \mathrm{CH}), 129.4(2 \times$ $\mathrm{CH}), 128.3(2 \times \mathrm{CH}), 127.0\left(\mathrm{CH}_{2}\right), 121.2(\mathrm{CH}), 115.9(2 \times \mathrm{CH}), 71.5(\mathrm{CH})$, 36.7 $\left(\mathrm{CH}_{2}\right), 19.7\left(\mathrm{CH}_{3}\right) \mathrm{ppm}$; GC RT $16.85 \mathrm{~min}$; LRMS (EI) $\mathrm{m} / \mathrm{z}(\%)=302$ $\left(\mathrm{M}^{+}, 9\right), 210$ (13), 209 (100), 143 (93), 125 (35), 121 (21), 94 (47), 77 (34), 67 (26), 65 (11); HRMS (EI) Calcd. for $\mathrm{C}_{17} \mathrm{H}_{18} \mathrm{O}_{3} \mathrm{~S}$ 302.0977, found 302.0985

(4-phenoxypent-1-en-2-yl)benzene (3ag): Compound 3ag was prepared from $2 \mathrm{~g}$ after $16 \mathrm{~h}$, following the general procedure. It was purified by FC (100\% Hexane to $95: 5$ Hexane/EtOAc) and obtained as a colorless oil (24 $\mathrm{mg}, 0.10 \mathrm{mmol}, 40 \%$ ): TLC Rf 0.77 (95:5 Hexane/EtOAc); IR v 2972, 2949 , 2930, 2911, 1598, 1493, 1375, 1231, 1174, 1081, 1067, 1054, 912, 781, $750,713,692 \mathrm{~cm}^{-1} ;{ }^{1} \mathrm{H}-\mathrm{NMR}\left(300 \mathrm{MHz}, \mathrm{CDCl}_{3}\right) \delta 7.45-7.16(\mathrm{~m}, 7 \mathrm{H}), 6.94$ - $6.85(\mathrm{~m}, 1 \mathrm{H}), 6.84-6.74(\mathrm{~m}, 2 \mathrm{H}), 5.33(\mathrm{~d}, J=1.4 \mathrm{~Hz}, 1 \mathrm{H}), 5.18(\mathrm{~d}, J=$ $1.1 \mathrm{~Hz}, 1 \mathrm{H}), 4.45-4.38(\mathrm{~m}, 1 \mathrm{H}), 3.08$ (ddd, $J=14.1,5.8,0.9 \mathrm{~Hz}, 1 \mathrm{H}), 2.65$ (ddd, $J=14.1,7.4,0.9 \mathrm{~Hz}, 1 \mathrm{H}), 1.28(\mathrm{~d}, J=6.1 \mathrm{~Hz}, 3 \mathrm{H}) \mathrm{ppm} ;{ }^{13} \mathrm{C}-\mathrm{NMR}$ $\left(101 \mathrm{MHz}, \mathrm{CDCl}_{3}\right) \delta 157.9(\mathrm{C}), 145.3(\mathrm{C}), 141.2(\mathrm{C}), 129.5(2 \times \mathrm{CH}), 128.5$ $(2 \times \mathrm{CH}), 127.7(\mathrm{CH}), 126.4(2 \times \mathrm{CH}), 120.8(\mathrm{CH}), 116.2(2 \times \mathrm{CH}), 115.5$ $(\mathrm{CH}), 72.5(\mathrm{CH}), 42.7\left(\mathrm{CH}_{2}\right), 19.7\left(\mathrm{CH}_{3}\right) \mathrm{ppm}$; GC $R_{T} 11.33 \mathrm{~min}$; LRMS (EI) $\mathrm{m} / \mathrm{z}(\%)=238\left(\mathrm{M}^{+}, 25\right), 146(12), 145(100), 144(30), 143(18), 130(17)$, 129 (84), 128 (21), 121 (54), 117 (21), 115 (18), 103 (33), 94 (17), 91 (22), 77 (34), 65 (10); HRMS (EI) Calcd. for $\mathrm{C}_{17} \mathrm{H}_{18} \mathrm{O} 238.1358$, found 238.1361.

2-methylene-3-phenethyl-4-phenoxypentanenitrile (3ah): Compound $2 \mathrm{ah}$ was prepared from $\mathbf{2 h}$ after $48 \mathrm{~h}$, following the general procedure. In this case 2 equiv of $1 \mathrm{a}$ and 1 equiv of $\mathbf{2 h}$ were used. It was purified by FC (100\% Hexane to $95: 5 \mathrm{Hexane} / \mathrm{EtOAc}$ ) and obtained as a colorless oil (26 $\mathrm{mg}, 0.09 \mathrm{mmol}, 32 \%$ in a $3: 2 \mathrm{dr}$ according to GC): TLC $R_{f} 0.30$ (95:5 Hexane/EtOAc); IR $v$ 2950, 2925, 2166, 2036, 1598, 1492, 1238, 1078 944, 752, $694 \mathrm{~cm}^{-1} ;{ }^{1} \mathrm{H}-\mathrm{NMR} \quad\left(300 \mathrm{MHz}, \mathrm{CDCl}_{3}\right.$ ) (mixture of diastereoisomers) $\delta 7.35-7.13(\mathrm{~m}, 9 \mathrm{H}), 7.00-6.84(\mathrm{~m}, 3 \mathrm{H}), 6.10(\mathrm{~d}, J=$ $0.6 \mathrm{~Hz}, 0.47 \mathrm{H}$ ), 6.08 (d, $J=0.5 \mathrm{~Hz}, 0.44 \mathrm{H}$ ), 5.84 (brs, $0.47 \mathrm{H}$ ), 5.82 (brs, $0.47 \mathrm{H}), 4.45-4.33(\mathrm{~m}, 1 \mathrm{H}), 2.84-2.68(\mathrm{~m}, 1 \mathrm{H}), 2.61-2.36(\mathrm{~m}, 2 \mathrm{H}), 2.28$ $-2.12(\mathrm{~m}, 1 \mathrm{H}), 2.08-1.80(\mathrm{~m}, 2 \mathrm{H}), 1.30(\mathrm{~d}, J=0.7 \mathrm{~Hz}, 1.5 \mathrm{H}), 1.28(\mathrm{~d}, J=$ $0.6 \mathrm{~Hz}, 1.5 \mathrm{H}$ ) ppm; ${ }^{13} \mathrm{C}-\mathrm{NMR} \quad\left(101 \mathrm{MHz}, \mathrm{CDCl}_{3}\right.$ ) (mixture of diastereoisomers) $\delta 157.7,157.6,141.08,141.05,133.9,133.7,129.8$, $129.7,128.67,128.65,128.5,126.3,126.28,121.4,121.3,116.3,116.0$, $75.1,75.0,50.31,50.26,33.31,33.3,30.9,17.5,17.4 \mathrm{ppm} ; \mathrm{GC}$ Diastereoisomer A: $R_{T} 15.90 \mathrm{~min}$; Diastereoisomer $B: R_{T} 16.00 \mathrm{~min}$; LRMS (EI) Diastereoisomer $A: m / z(\%)=291\left(\mathrm{M}^{+}, 15\right), 121(83), 105(15), 94(68)$, 92 (11), 91 (100), 77 (25), 65 (13); Diastereoisomer B: $m / z(\%)=291\left(\mathrm{M}^{+}\right.$, 14), 121 (81), 105 (16), 94 (73), 91 (100), 77 (23), 65 (14); HRMS (EI) Calcd. for $\mathrm{C}_{20} \mathrm{H}_{21} \mathrm{NO} 291.1623$, found 291.1623.

General procedure for the decarboxylative arylation of carboxylic acids: 2-(phenylsulfonyl)benzo[d]thiazole $(4,68.8 \mathrm{mg}, 0.25 \mathrm{mmol}, 1$ equiv) was added to a 2 dram vial equipped with a stirring magnetic bar, followed by RFTA ( $13.6 \mathrm{mg}, 0.025 \mathrm{mmol}, 10 \mathrm{~mol}-\%)$, the corresponding carboxylic acid $(0.50 \mathrm{mmol}, 2$ equiv) and $\mathrm{MeCN}(2.5 \mathrm{~mL})$. The vial was sealed with a septum and the reaction mixture submitted to three cycles of freeze-pumpthaw. Finally, the vial was equipped with an $\mathrm{Ar}$ balloon and the yellow mixture ${ }^{[38]}$ was stirred at $25^{\circ} \mathrm{C}$ under blue LEDs irradiation $(\lambda=455 \mathrm{~nm}$, $\left.15 \pm 2 \mathrm{~mW} / \mathrm{cm}^{2}\right)^{[34]}$ until no progress was observed by TLC or GC (generally $36 \mathrm{~h}$ ). The solvent was removed under reduced pressure, affording a residue which was purified by FC.

2-(1-phenoxyethyl)benzo[ $[\mathrm{d}]$ thiazole (5a): Compound $5 \mathrm{a}$ was prepared from 2-Phenoxypropanoic acid (1a) following the general procedure for the decarboxylative arylation. It was purified by FC (100\% Hexane to $90: 10$
Hexane/EtOAc) and obtained as a white solid (38 $\mathrm{mg}, 0.15 \mathrm{mmol}, 60 \%$ ): TLC $R_{f} 0.47$ (95:5 Hexane/EtOAc); IR v 2987, 2901, 1597, 1493, 1261 1217, 1065, 907, 753, $728 \mathrm{~cm}^{-1} ;{ }^{1} \mathrm{H}-\mathrm{NMR}\left(400 \mathrm{MHz}, \mathrm{CDCl}_{3}\right) \delta 8.02(\mathrm{~d}, J=$ $8.1 \mathrm{~Hz}, 1 \mathrm{H}), 7.85(\mathrm{~d}, J=8.0 \mathrm{~Hz}, 1 \mathrm{H}), 7.53-7.45(\mathrm{~m}, 1 \mathrm{H}), 7.42-7.35(\mathrm{~m}$, $1 \mathrm{H}), 7.29-7.22(\mathrm{~m}, 2 \mathrm{H}), 7.03-6.98(\mathrm{~m}, 2 \mathrm{H}), 6.98-6.93(\mathrm{~m}, 1 \mathrm{H}), 5.77(\mathrm{q}$ $J=6.5 \mathrm{~Hz}, 1 \mathrm{H}), 1.85(\mathrm{~d}, J=6.5 \mathrm{~Hz}, 3 \mathrm{H}) \mathrm{ppm} ;{ }^{13} \mathrm{C}-\mathrm{NMR}\left(101 \mathrm{MHz}, \mathrm{CDCl}_{3}\right)$ $175.1(\mathrm{C}), 157.4(\mathrm{C}), 152.9(\mathrm{C}), 134.9(\mathrm{C}), 129.7(2 \times \mathrm{CH}), 126.3(\mathrm{CH})$, $125.3(\mathrm{CH}), 123.1(\mathrm{CH}), 122.1(\mathrm{CH}), 121.9(\mathrm{CH}), 115.9(2 \times \mathrm{CH}), 74.7(\mathrm{CH})$, $23.0\left(\mathrm{CH}_{3}\right)$ ppm; GC RT $14.96 \mathrm{~min}$; LRMS (EI) $\mathrm{m} / z(\%)=255\left(\mathrm{M}^{+}, 3\right), 163$ (11), 162 (100), 109 (14); HRMS (EI) Calcd. for $\mathrm{C}_{15} \mathrm{H}_{13} \mathrm{NOS} 255.0718$, found 255.0706 .

\section{2-(2,3-dihydrobenzo[b][1,4]dioxin-2-yl)benzo[ $d]$ thiazole}

(5b): Compound $\mathbf{5 b}$ was prepared from 1,4-Benzodioxane-2-carboxylic acid (1b) following the general procedure for the decarboxylative arylation. It was purified by FC (100\% Hexane to $95: 5$ Hexane/EtOAc) and obtained as a white solid (43 mg, $0.16 \mathrm{mmol}, 64 \%)$ : TLC $R_{f} 0.31$ (95:5 Hexane/EtOAc); IR v 2987, 2901, 1494, 1263, 1215, 1074, 1053, 907, 748, $729 \mathrm{~cm}^{-1} ;{ }^{1} \mathrm{H}$-NMR $\left(400 \mathrm{MHz}, \mathrm{CDCl}_{3}\right) \delta 8.06(\mathrm{~d}, J=8.2 \mathrm{~Hz}, 1 \mathrm{H}), 7.91(\mathrm{~d}, J$ $=8.0 \mathrm{~Hz}, 1 \mathrm{H}), 7.55-7.90(\mathrm{~m}, 1 \mathrm{H}), 7.45-7.39(\mathrm{~m}, 1 \mathrm{H}), 7.12-7.04(\mathrm{~m}, 1 \mathrm{H})$, $6.99-6.89(\mathrm{~m}, 3 \mathrm{H}), 5.65(\mathrm{dd}, J=6.9,2.7 \mathrm{~Hz}, 1 \mathrm{H}), 4.72(\mathrm{dd}, J=11.4,2.7$ $\mathrm{Hz}, 1 \mathrm{H}), 4.43(\mathrm{dd}, J=11.4,6.9 \mathrm{~Hz}, 1 \mathrm{H}) \mathrm{ppm} ;{ }^{13} \mathrm{C}-\mathrm{NMR}\left(101 \mathrm{MHz}, \mathrm{CDCl}_{3}\right)$ $\delta 167.8(\mathrm{C}), 153.1(\mathrm{C}), 143.2(\mathrm{C}), 142.4(\mathrm{C}), 135.0(\mathrm{C}), 126.5(\mathrm{CH}), 125.6$ $(\mathrm{CH}), 123.4(\mathrm{CH}), 122.4(\mathrm{CH}), 122.2(\mathrm{CH}), 122.0(\mathrm{CH}), 117.7(\mathrm{CH}), 117.6$ (CH), $73.6(\mathrm{CH}), 67.2\left(\mathrm{CH}_{2}\right) \mathrm{ppm}$; GC RT $16.66 \mathrm{~min}$; LRMS (EI) $\mathrm{m} / \mathrm{z}(\%)=$ $270\left(\mathrm{M}^{+}+1,11\right), 269(63), 241$ (11), $226(11), 224(36), 207$ (11), $162(12)$, 161 (100), 160 (37), 135 (26), 108 (12), 69 (11). Characterization data matched that reported in the literature. ${ }^{[39]}$

2-((2-methoxyphenoxy)methyl)benzo[d]thiazole (5c): Compound 5c was prepared from 2-(2-Methoxyphenoxy)acetic acid (1g) following the general procedure for the decarboxylative arylation. It was purified by FC (100\% Hexane to $85: 15$ Hexane/EtOAc) and obtained as a yellow oil (41 $\mathrm{mg}, 0.15 \mathrm{mmol}, 60 \%$ ): TLC $R_{f} 0.16$ (95:5 Hexane/EtOAc); IR v 2971, 2901 $1501,1254,1045,1028,905,760,724 \mathrm{~cm}^{-1} ;{ }^{1} \mathrm{H}-\mathrm{NMR}$ (300 MHz, $\left.\mathrm{CDCl}_{3}\right)$ $\delta 8.03(\mathrm{~d}, J=8.0 \mathrm{~Hz}, 1 \mathrm{H}), 7.89(\mathrm{~d}, J=8.6 \mathrm{~Hz}, 1 \mathrm{H}), 7.53-7.45(\mathrm{~m}, 1 \mathrm{H})$, $7.44-7.36(\mathrm{~m}, 1 \mathrm{H}), 7.06-7.00(\mathrm{~m}, 1 \mathrm{H}), 6.98-6.92(\mathrm{~m}, 2 \mathrm{H}), 6.91-6.83$ $(\mathrm{m}, 1 \mathrm{H}), 5.56(\mathrm{~s}, 2 \mathrm{H}), 3.92(\mathrm{~s}, 3 \mathrm{H}) \mathrm{ppm} ;{ }^{13} \mathrm{C}-\mathrm{NMR}\left(101 \mathrm{MHz}, \mathrm{CDCl}_{3}\right) \delta$ $150.1(\mathrm{C}), 147.4(\mathrm{C}), 126.6(\mathrm{C}), 125.7(\mathrm{C}), 123.2(\mathrm{CH}), 122.9(\mathrm{CH}), 122.1$ $(\mathrm{CH}), 121.1(2 \times \mathrm{CH}), 115.6(\mathrm{C}), 112.5(2 \times \mathrm{CH}), 69.3\left(\mathrm{CH}_{2}\right), 56.2\left(\mathrm{CH}_{3}\right)$ ppm; GC $R_{T} 16.44 \mathrm{~min}$; LRMS (EI) $\mathrm{m} / z(\%)=271\left(\mathrm{M}^{+}, 22\right), 149(13), 148$ (100); HRMS (EI) Calcd. for $\mathrm{C}_{15} \mathrm{H}_{13} \mathrm{NO}_{2} \mathrm{~S} 271.0667$, found 271.0667 .

2-(1-(benzyloxy)ethyl)benzo[d]thiazole (5d): Compound 5d was prepared from $(R)-(+)-2-($ Benzyloxy)propanoic acid (1i) following the general procedure for the decarboxylative arylation. It was purified by FC (100\% Hexane to $90: 10$ Hexane/EtOAc) and obtained as a pale-yellow oil (27 mg, $0.10 \mathrm{mmol}, 40 \%)$ : TLC $R_{f} 0.31$ (95:5 Hexane/EtOAc); IR v 2987, 2901, 1508, 1395, 1215, 1066, 1056, 907, 747, $725 \mathrm{~cm}^{-1} ;{ }^{1} \mathrm{H}-\mathrm{NMR}(300$ $\left.\mathrm{MHz}, \mathrm{CDCl}_{3}\right) \delta 8.03(\mathrm{dd}, J=8.1,0.5 \mathrm{~Hz}, 1 \mathrm{H}), 7.92(\mathrm{dd}, J=7.9,0.7 \mathrm{~Hz}$, $1 \mathrm{H}), 7.54-7.28(\mathrm{~m}, 8 \mathrm{H}), 5.01(\mathrm{q}, J=6.5 \mathrm{~Hz}, 1 \mathrm{H}), 4.69(\mathrm{~d}, J=11.6 \mathrm{~Hz}, 1 \mathrm{H})$, $4.60(\mathrm{~d}, J=11.6 \mathrm{~Hz}, 1 \mathrm{H}), 1.70(\mathrm{~d}, J=6.5 \mathrm{~Hz}, 3 \mathrm{H}) \mathrm{ppm} ;{ }^{13} \mathrm{C}-\mathrm{NMR}(101$ $\left.\mathrm{MHz}, \mathrm{CDCl}_{3}\right) \delta 176.5(\mathrm{C}), 152.8(\mathrm{C}), 137.6(\mathrm{C}), 134.9(\mathrm{C}), 128.6(2 \times \mathrm{CH})$, $128.1(2 \times \mathrm{CH}), 128.0(2 \times \mathrm{CH}), 126.2(\mathrm{CH}), 125.3(\mathrm{CH}), 123.0(\mathrm{CH}), 122.1$ $(\mathrm{CH}), 75.7(\mathrm{CH}), 71.8\left(\mathrm{CH}_{2}\right), 22.7\left(\mathrm{CH}_{3}\right) \mathrm{ppm}$; GC RT $15.58 \mathrm{~min} ; \mathrm{LRMS}$ (EI) $m / z(\%)=224$ (10), 164 (12), 163 (100), $162\left(\mathrm{M}^{+}-\right.$OBn, 46), 91 (33); HRMS (EI) Calcd. for $\mathrm{C}_{9} \mathrm{H}_{9} \mathrm{NS} 163.0456$, found 163.0451 .

\section{2-((3aR,6R,6aR)-6-methoxy-2,2-dimethyltetrahydrofuro[3,4} d] [1,3]dioxol-4-yl)benzo[d]thiazole (5e): Compound $5 \mathbf{e}$ was prepared from 2,3-O-isopropylidene-1-O-methyl-D-ribosic acid (1h) following the general procedure for the decarboxylative arylation. It was purified by FC (100\% Hexane to $90: 10$ Hexane/EtOAc) and obtained as a colorless oil (28 mg, 0.09 mmol, 35\%): TLC Rf 0.23 (95:5 Hexane/EtOAc); IR v 2986, 
2934, 1598, 1493, 1446, 1375, 1306, 1239, 1141, 1084, 907, 867, 728, $689 \mathrm{~cm}^{-1} ;{ }^{1} \mathrm{H}-\mathrm{NMR}\left(300 \mathrm{MHz}, \mathrm{CDCl}_{3}\right) \delta 8.02(\mathrm{~d}, J=8.2 \mathrm{~Hz}, 1 \mathrm{H}), 7.88(\mathrm{dd}$, $J=7.9,0.7 \mathrm{~Hz}, 1 \mathrm{H}), 7.52-7.44(\mathrm{~m}, 1 \mathrm{H}), 7.43-7.35(\mathrm{~m}, 1 \mathrm{H}), 5.60$ (dd, $J=$ $5.9,1.1 \mathrm{~Hz}, 1 \mathrm{H}), 5.54(\mathrm{br} \mathrm{s}, 1 \mathrm{H}), 5.17(\mathrm{~s}, 1 \mathrm{H}), 4.69(\mathrm{~d}, J=5.9 \mathrm{~Hz}, 1 \mathrm{H}), 3.39$ (s, 3H), 1.57 (s, 3H), 1.57 (s, 3H) ppm; ${ }^{13} \mathrm{C}-\mathrm{NMR}\left(75 \mathrm{MHz}, \mathrm{CDCl}_{3}\right) \delta 170.2$ (C), $152.9(\mathrm{C}), 135.4(\mathrm{C}), 126.3(\mathrm{CH}), 125.4(\mathrm{CH}), 123.3(\mathrm{CH}), 121.8(\mathrm{CH})$, $113.1(\mathrm{C}), 111.1(\mathrm{CH}), 86.4(\mathrm{CH}), 85.4(\mathrm{CH}), 84.2(\mathrm{CH}), 56.2\left(\mathrm{CH}_{3}\right), 26.7$ $\left(\mathrm{CH}_{3}\right), 25.3\left(\mathrm{CH}_{3}\right)$ ppm; GC RT $15.48 \mathrm{~min}$; LRMS (EI) $\mathrm{m} / z(\%)=292\left(\mathrm{M}^{+}\right.$. $\left.\mathrm{CH}_{3}, 10\right), 218$ (11), 208 (11), 207 (92), 190 (25), 189 (23), 165 (14), 164 (39), 163 (30), 162 (17), 161 (100), 160 (22), 135 (25), 108 (12), 85 (15), 58 (14); HRMS (El) Calcd. for $\mathrm{C}_{15} \mathrm{H}_{17} \mathrm{NO}_{4} \mathrm{~S} 307.0878$, found 307.0883.

$\mathbf{N}$-(benzo[ $[\boldsymbol{d}]$ thiazol-2-ylmethyl)aniline (5f): Compound $\mathbf{5 f}$ was prepared from $(M)$-Phenylglycine (11) following the general procedure for the decarboxylative arylation. It was purified by FC $(100 \%$ Hexane to $80: 20$ Hexane/EtOAc) and then treated with $\mathrm{PrOH} / \mathrm{Hexanes}$ to filtered out the starting sulfone and obtain $\mathbf{5 f}$ as a colorless oil ( $36 \mathrm{mg}, 0.15 \mathrm{mmol}, 60 \%$ ): TLC $R_{f} 0.13$ (95:5 Hexane/EtOAc); IR $v$ 2987, 2901, 1216, 1066, 1056, $906,754,727,671 \mathrm{~cm}^{-1} ;{ }^{1} \mathrm{H}-\mathrm{NMR}\left(300 \mathrm{MHz}, \mathrm{CDCl}_{3}\right) \delta 8.01(\mathrm{~d}, J=7.9 \mathrm{~Hz}$, $1 \mathrm{H}), 7.83(\mathrm{dd}, J=8.0,0.6 \mathrm{~Hz}, 1 \mathrm{H}), 7.51-7.45(\mathrm{~m}, 1 \mathrm{H}), 7.39-7.34(\mathrm{~m}, 1 \mathrm{H})$, 7.23 - $7.15(\mathrm{~m}, 2 \mathrm{H}), 6.84-6.67(\mathrm{~m}, 3 \mathrm{H}), 4.78(\mathrm{~s}, 2 \mathrm{H}) \mathrm{ppm} ;{ }^{13} \mathrm{C}-\mathrm{NMR}(101$ $\left.\mathrm{MHz}, \mathrm{CDCl}_{3}\right) \delta 173.2(\mathrm{C}), 153.6(\mathrm{C}), 147.0(\mathrm{C}), 129.5(2 \times \mathrm{CH}), 126.1(\mathrm{CH})$, $125.0(\mathrm{CH}), 122.9(\mathrm{CH}), 121.9(\mathrm{CH}), 118.9(\mathrm{C}), 113.4(2 \times \mathrm{CH}), 47.2\left(\mathrm{CH}_{2}\right)$ ppm; GC $R_{T} 16.29 \mathrm{~min} ;$ LRMS (El) $\mathrm{m} / \mathrm{z}(\%)=241\left(\mathrm{M}^{+}+1,18\right), 240(100)$, 239 (27), 238 (24), 237 (27), 210 (13), 149 (10), 148 (36), 136 (23), 135 (11), 108 (10), 106 (76), 105 (13), 104 (12), 77 (30); HRMS (EI) Calcd. for $\mathrm{C}_{14} \mathrm{H}_{12} \mathrm{~N}_{2} \mathrm{~S} 240.0721$, found 240.0718 .

General procedure for the synthesis of 3qa and 7: 2-(but-3-en-1yloxy)propanoic acid ${ }^{[40]}(1 \mathrm{q})(28.8 \mathrm{mg}, 0.2 \mathrm{mmol}, 1$ equiv) was added to a 2 dram vial equipped with a stirring magnetic bar, followed by RFTA ( 5.44 $\mathrm{mg}, 0.01 \mathrm{mmol}, 5 \mathrm{~mol}-\%)$, and a solution of $2 \mathrm{a}(0.22 \mathrm{mmol}, 56.4 \mathrm{mg}, 1.1$ equiv) in $\mathrm{MeCN}(4 \mathrm{~mL})$. The vial was sealed with a septum and the reaction mixture submitted to three cycles of freeze-pump-thaw. Finally, the vial was equipped with an Ar balloon and the yellow mixture was stirred at 25 ${ }^{\circ} \mathrm{C}$ under blue LEDs irradiation $\left(\lambda=455 \mathrm{~nm}, 15 \pm 2 \mathrm{~mW} / \mathrm{cm}^{2}\right)$ until no progress was observed by TLC or GC (24 h). The solvent was removed under reduced pressure and pure compounds $3 q a$ and 7 was obtained after purification by flash column chromatography.

Butyl 4-(but-3-en-1-yloxy)-2-methylenepentanoate (3qa): Compound 3qa was prepared from 2-(but-3-en-1-yloxy)propanoic acid (1q) following the procedure above described. It was purified by FC $(100 \%$ Hexane to $90: 10$ Hexane/EtOAc) and obtained as a colorless oil ( $15 \mathrm{mg}, 0.06 \mathrm{mmol}$, $30 \%)$ : TLC $R_{f} 0.75$ (90:10 Hexane/EtOAc); IR $v 2960,2930,2873,1716$, $1631,1458,1326,1219,1125,912,735 \mathrm{~cm}^{-1} ;{ }^{1} \mathrm{H}-\mathrm{NMR}\left(300 \mathrm{MHz}, \mathrm{CDCl}_{3}\right)$ $\delta 6.19(\mathrm{~d}, \mathrm{~J}=1.7 \mathrm{~Hz}, 1 \mathrm{H}), 5.81$ (ddt, $J=17.0,10.2,6.7 \mathrm{~Hz}, 1 \mathrm{H}$ ), 5.60 (dd, $J=2.7,1.1 \mathrm{~Hz}, 1 \mathrm{H}), 5.12-4.97(\mathrm{~m}, 2 \mathrm{H}), 4.15(\mathrm{t}, J=6.6 \mathrm{~Hz}, 2 \mathrm{H}), 3.64-$ $3.39(\mathrm{~m}, 3 \mathrm{H}), 2.59$ (ddd, $J=13.8,6.7,1.0 \mathrm{~Hz}, 1 \mathrm{H}), 2.35$ (ddd, $J=13.8,6.7$, $1.0 \mathrm{~Hz}, 1 \mathrm{H}), 2.32-2.25(\mathrm{~m}, 2 \mathrm{H}), 1.72-1.59(\mathrm{~m}, 2 \mathrm{H}), 1.50-1.35(\mathrm{~m}, 2 \mathrm{H})$, 1.14 (d, $J=6.2 \mathrm{~Hz}, 3 \mathrm{H}), 0.95$ (t, $J=7.3 \mathrm{~Hz}, 3 \mathrm{H}) \mathrm{ppm} ;{ }^{13} \mathrm{C}-\mathrm{NMR}(101 \mathrm{MHz}$, $\left.\mathrm{CDCl}_{3}\right) \delta 167.5(\mathrm{C}), 137.9(\mathrm{C}), 135.6(\mathrm{CH}), 127.2\left(\mathrm{CH}_{2}\right), 116.3\left(\mathrm{CH}_{2}\right), 74.3$ $(\mathrm{CH}), 68.2\left(\mathrm{CH}_{2}\right), 64.7\left(\mathrm{CH}_{2}\right), 39.4\left(\mathrm{CH}_{2}\right), 34.7\left(\mathrm{CH}_{2}\right), 30.8\left(\mathrm{CH}_{2}\right), 19.8$ $\left(\mathrm{CH}_{2}\right), 19.4\left(\mathrm{CH}_{3}\right), 13.9\left(\mathrm{CH}_{3}\right) \mathrm{ppm}$; GC $R T 11.23 \mathrm{~min} ; \mathrm{LRMS}(\mathrm{El}) \mathrm{m} / \mathrm{z}(\%)$ $=155\left(\mathrm{M}^{+}-\mathrm{C}_{5} \mathrm{H}_{9} \mathrm{O}, 11\right), 113(45), 111(11), 99(54), 95$ (18), 81 (31), 69 (18), 67 (14), 55 (100); HRMS (EI) Calcd. for $\mathrm{C}_{14} \mathrm{H}_{24} \mathrm{O}_{3}-\mathrm{C}_{9} \mathrm{H}_{5} \mathrm{O}_{2} 155.1072$, found 155.1066 .

Butyl

2-methylene-4-[(2R,3S)-2-methyltetrahydrofuran-3yl)butanoate (7): Compound 7 was prepared from 2-(but-3-en-1yloxy)propanoic (1q) following the procedure above described. It was purified by FC (100\% Hexane to $90: 10$ Hexane/EtOAc) and obtained as a colorless oil ( $10 \mathrm{mg}, 0.04 \mathrm{mmol}, 21 \%)$ : TLC Rf 0.44 (90:10 Hexane/EtOAc); IR v 2959, 2929, 2872, 1718, 1457, 1186, 1155, 1069, 941, $735 \mathrm{~cm}^{-1} ;{ }^{1} \mathrm{H}-$
NMR $\left(300 \mathrm{MHz}, \mathrm{CDCl}_{3}\right) \delta 6.15(\mathrm{~d}, J=1.4 \mathrm{~Hz}, 1 \mathrm{H}), 5.54(\mathrm{dd}, J=2.7,1.3$ $\mathrm{Hz}, 1 \mathrm{H}), 4.16(\mathrm{t}, J=6.6 \mathrm{~Hz}, 2 \mathrm{H}), 4.07(\mathrm{p}, J=6.5 \mathrm{~Hz}, 1 \mathrm{H}), 3.93(\mathrm{td}, J=8.4$, $3.7 \mathrm{~Hz}, 1 \mathrm{H}), 3.69(\mathrm{dd}, J=15.6,8.3 \mathrm{~Hz}, 1 \mathrm{H}), 2.45-2.20(\mathrm{~m}, 2 \mathrm{H}), 2.19-$ $1.95(\mathrm{~m}, 2 \mathrm{H}), 1.75-1.57(\mathrm{~m}, 5 \mathrm{H}), 1.47-1.36(\mathrm{~m}, 2 \mathrm{H}), 1.08(\mathrm{~d}, J=6.5 \mathrm{~Hz}$ $3 \mathrm{H}), 0.95(\mathrm{t}, J=7.3 \mathrm{~Hz}, 3 \mathrm{H}) \mathrm{ppm} ;{ }^{13} \mathrm{C}-\mathrm{NMR}\left(101 \mathrm{MHz}, \mathrm{CDCl}_{3}\right) \delta 167.4$, $141.0,124.7,66.5,64.7,41.8,31.11,31.08,30.8,28.9,19.4,16.2,13.9$ ppm; GC $R_{T} 12.61 \mathrm{~min}$; LRMS (EI) $\mathrm{m} / \mathrm{z}(\%)=167\left(\mathrm{M}^{+}, 25\right), 166(84), 151$ (48), 140 (18), 139 (10), $138(20), 125$ (20), 124 (16), 123 (39), $122(20)$, 121 (26), 112 (16), 111 (54), 110 (17), 109 (11), 108 (11), 107 (11), 105 (10), 99 (11), 98 (28), 97 (66), 96 (25), 95 (100), 94 (40), 93 (30); HRMS (EI) Calcd. for $\mathrm{C}_{14} \mathrm{H}_{24} \mathrm{O}_{3}-\mathrm{C}_{4} \mathrm{H}_{10} \mathrm{O}_{2}$ 166.0994, found 166.099.

Butyl 2-((2,3-dihydrobenzo[b][1,4]dioxin-2-yl)methyl)-4-( $\quad$ tolyloxy)butanoate (6): Compound $1 \mathbf{d}(57 \mathrm{mg}, 0.3 \mathrm{mmol}, 1.5$ equiv.) was added to a 2 dram vial equipped with a stirring magnetic bar, followed by a solution of 3 ba $\left(49.8 \mathrm{mg}, 0.2 \mathrm{mmol}, 1\right.$ equiv.) in $\mathrm{MeCN}(0.7 \mathrm{~mL}), \mathrm{Na}_{2} \mathrm{CO}_{3}$ (4.24 mg, $0.04 \mathrm{mmol}, 20 \mathrm{~mol}-\%)$ and [Mes-Acr] $\mathrm{ClO}_{4}(2.08 \mathrm{mg}, 2.5 \mathrm{~mol}-\%)$. Finally, $\mathrm{H}_{2} \mathrm{O}(0.3 \mathrm{~mL})$ was added and the vial was sealed with a cap. The yellow solution was irradiated using blue LED's and stirred at room temperature, without any inert atmosphere, until complete conversion was observed (monitored by TLC and/or GC, $22 \mathrm{~h}$ ). The reaction mixture was concentrated under reduced pressure and the residue was purified by FC (100\% Hexane to $90: 10$ Hexane/EtOAc) to obtain 6 as a colorless oil (36 mg, $0.09 \mathrm{mmol}$, 45\%): ( 2:1 dr according to GC-MS); TLC $R_{f}$ 0.47(90:10 Hexane/EtOAc); IR v 2965, 2926, 1729, 1510, 1494, 1264, 1241, 1175, $1045,817,746 \mathrm{~cm}^{-1}$; ${ }^{1} \mathrm{H}-\mathrm{NMR}\left(400 \mathrm{MHz}, \mathrm{CDCl}_{3}\right)$ (mixture of diastereoisomers) $\delta 7.07(\mathrm{~d}, J=8.2 \mathrm{~Hz}, 2 \mathrm{H}), 6.85-6.82(\mathrm{~m}, 4 \mathrm{H}), 6.78$ $6.76(\mathrm{~m}, 2 \mathrm{H}), 4.25-3.87(\mathrm{~m}, 6 \mathrm{H}), 3.11-2.85(\mathrm{~m}, 1 \mathrm{H}), 2.28(\mathrm{~s}, 3 \mathrm{H}), 2.21$ $1.93(\mathrm{~m}, 2 \mathrm{H}), 1.88-1.74(\mathrm{~m}, 1 \mathrm{H}), 1.60-1.55(\mathrm{~m}, 2 \mathrm{H}), 1.41-1.30(\mathrm{~m}, 2 \mathrm{H})$, $0.94-0.77(\mathrm{~m}, 3 \mathrm{H}) \mathrm{ppm} ;{ }^{13} \mathrm{C}-\mathrm{NMR}\left(101 \mathrm{MHz}, \mathrm{CDCl}_{3}\right)$ (mixture of diastereoisomers) $\delta 175.3,156.7,143.24,143.15,143.12,130.20,130.19$, 130.0, 121.7, 121.6, 121.48, 117.52, 117.51, 117.2, 114.53, 114.50, 71.3, $68.1,67.8,65.7,65.6,64.84,64.81,39.0,38.6,33.4,33.2,32.5,31.7,30.7$, 20.6, 19.29, 19.28, 13.83,13.80 ppm; GC RT Diastereoisomer A: 24.07 $\mathrm{min}$; Diastereoisomer B: $24.50 \mathrm{~min}$ LRMS (EI) $\mathrm{m} / \mathrm{z}(\%)=$ Diastereoisomer A: $398\left(\mathrm{M}^{+}, 10\right), 325$ (13), 291 (30), 236 (15), 235 (100), 149 (12), $147(10)$, 135 (26), 125 (10), 121 (17), 108 (10), 107 (11), 101 (10); Diastereoisomer B: $398\left(\mathrm{M}^{+}, 11\right), 325$ (13), 291 (27), 236 (14), 235 (100), 149 (11), 135 (22), 121 (15); HRMS (EI) Calcd. for $\mathrm{C}_{24} \mathrm{H}_{30} \mathrm{O}_{5} 398.2093$, found 398.2081.

\section{Acknowledgments}

This work was generously supported by the Spanish Ministerio de Economía y Competitividad (CTQ2017-88171-P) and the Generalitat Valenciana (AICO/2017/007). N. P. R. thanks to Instituto de Síntesis Orgánica for financial support.

Keywords: allylation $\bullet$ carboxylic acids $\bullet$ photocatalysis $\bullet$ radicals - sustainable chemistry

[1] For recent reviews, see: a) N. A. Romero, D. A. Nicewicz, Chem. Rev. 2016, 116, 10075-10166; b) D. Staveness, I. Bosque; C. R. J. Stephenson, Acc. Chem. Res., 2016, 49, 2295-2306; c) J. K. Matsui, S B. Lang, D. R. Heitz, G. A. Molander, ACS Catal. 2017, 7, 2563-2575; d) L. M. Marzo, S. K. Pagire, O. Reiser, B. König, Angew. Chem. Int. Ed. 2018, 57, 10034-10072; e) C. R. J. Stephenson, T. P. Yoon, D. W. C. Macmillan, Visible Light Photocatalysis in Organic Chemistry; Wiley: Weinheim, Germany, 2018.

[2] J. K. Kochi, T. M. Bockman, S. M. Hubig, J. Org. Chem. 1997, 62, 2210 2221. 
[3] Potassium alkyltrifluoroborates have relatively low oxidation potentials and have been employed as radical precursors in many photocatalystmediated transformations. For some relevant examples, see: a) Y. Yasu, T. Koike, M. Akita, Adv. Synth. Catal. 2012, 354, 3414-3420; b) K. Miyazawa, Y. Yasu, T. Koike, M. Akita, Chem. Commun. 2013, 49, 72497251; c) J. C. Tellis, D. N. Primer, G. A. Molander, Science 2014, 345 433-436; d) G. A. Molander, J. Org. Chem. 2015, 80, 7837-7848; e) D. N. Primer, I. Karakaya, J. C. Tellis, G. A. Molander, J. Am. Chem. Soc 2015, 137, 2195-2198.

[4] Light-mediated oxidation of alkyl bis(catecholato)-silicates can be carried out using mildly oxidizing photocatalysts to generate alkyl radicals $\left(E_{1 / 2}\right.$ $=+0.3$ to $+0.9 \mathrm{~V}$ vs. SCE). For relevant references, see: a) D. Matsuoka Y. Nishigaichi, Chem. Lett. 2015, 44, 163-165; b) V. Corcé, L. M. Chamoreau, E. Derat, J.-P. Goddard, C. Ollivier, L. Fensterbank, Angew. Chem., Int. Ed. 2015, 54, 11414-11418; c) K. Lin, C. B. Kelly, M. Jouffroy, G. A. Molander, Org. Synth. 2017, 94, 16-33.

[5] DHPs might undergo photocatalyst-mediated oxidative fragmentation $\left(E_{1 / 2}=+1.10 \mathrm{~V} v s\right.$. SCE $)$, generating alkyl radicals and pyridinium salts. See: J.-P. Cheng, Y. Lu, X.-Q. Zhu, Y. Sun, F. Bi, J. He, J. Org. Chem. 2000, 65, 3853-3857.

[6] Single-electron reduction of Katritzky pyridinium salts $\left(E_{1 / 2}=-0.93 \mathrm{~V} v\right.$. SCE) has recently been employed for the generation of alkyl radicals. See: F. J. R. Klauck, M. J. James, F. Glorius, Angew. Chem., Int. Ed., 2017, 56, 12336-12339.

[7] For recent reviews, see: a) A. J. J. Straathof, Chem. Rev. 2014, 114, 1871-1908; b) H. Fu, Y. Jin, Asian J. Org. Chem. 2017, 6, 368 - 385.

[8] L. Capaldo, L. Buzzetti, D. Merli, M. Fagnoni, D. Ravelli, J. Org. Chem. 2016, 81, 7102-7109.

[9] For some relevant examples, see: a) D. S. Hamilton, D. A. Nicewicz, J. Am. Chem. Soc. 2012, 134, 18577-18580; b) A. J. Perkowski, D. A Nicewicz, J. Am. Chem. Soc. 2013, 135, 10334-10337; c) M. A. Zeller, M. Riener, D. A. Nicewicz, Org. Lett. 2014, 16, 4810-4813.

[10] a) D. Hanss, J. C. Freys, G. Bernardinelli, O. S. Wenger, Eur. J. Inorg Chem. 2009, 4850-4859; b)This photocatalyst can be purchased from Sigma-Aldrich (Product number: 804215 ) at $\approx 1360 € / \mathrm{mmol}$.

[11] Riboflavin can be purchased from TCl-Europe (Product number: R0020) at $\approx 0.27 € / \mathrm{mmol}$.

[12] K. S. Conrad, C. C. Manahan, B. R. Crane, Nat. Chem. Bio. 2014, 10, 801-809.

[13] a) For photophysical properties of riboflavin, see: B. König, S. Kümmel, R. Cibulka, Flavin photocatalysis. In Chemical Photocatalysis: B. König, Ed. De Gruyter: Regensburg, 2013, p 45-66, and references therein; b) For standard redox potentials of carboxylic acids, see: H. G. Roth, N. A. Romero, D. A. Nicewicz, Synlett 2016, 27, 714-723.

[14] a) J. B. Metternich, R. Gilmour, J. Am. Chem. Soc. 2016, 138, 1040-1045; b) T. Morack, J. B. Metternich, R. Gilmour, Org. Lett. 2018, 20, 1316-1319.

[15] S. Bloom, C. Liu, D. K. Kölmel, J. X. Qiao, Y. Zhang, M. A. Poss, W. R. Ewing, D. W. C. MacMillan, Nat. Chem. 2018, 10, 205-211.

[16] N. P. Ramirez, B. König, J. C. Gonzalez-Gomez, Org. Lett, 2019, 21, 1368-1373.

[17] a) G. A. Russell, P. Ngoviwatchai, H. Tashtoush, A. Pla-Dalmau, R. K. Khanna, J. Am. Chem. Soc. 1988, 110, 3530-3538; b) Y. Amaoka, M. Nagatomo, M. Watanabe, K. Tao, S. Kamijo, M. Inoue, Chem. Sci. 2014, 5, 4339-4345; c) A. Noble, D. W. C. MacMillan, J. Am. Chem. Soc. 2014, 136, 11602-11605; d) Reference 4b.

[18] For precedents in the radical addition to 2-(phenylsulfonyl)benzothiazole, see: a) D. B. Jacobson, B. S. Freiser, J. Am. Chem. Soc. 1984, 106, 4623-4624; b) Y. Ikeda, R. Ueno, Y. Akai, E. Shirakawa, Chem. Commun., 2018, 54, 10471-10474.

[19] For a recent review: A. Rouf, C. Tanyeli, Eur. J. Med. Chem. 2015, 97, 911-927.

[20] C. Hu, Y. Chen, Org. Chem. Front., 2015, 2, 1352-1355.

[21] L. Cui, H. Chen, C. Liu, C. Li, Org. Lett., 2016, 18, 2188-2191.
[22] Y. Duan, M. Zhang, R. Ruzi, Z. Wu, C. Zhu, Org. Chem. Front., 2017, 4 525-528. This photocatalyst can be purchased from Strem Chemicals (Product number: $77-0465$ ) at $\approx 582 € / \mathrm{mmol}$. The reaction is limited to $\mathrm{N}$-arylglycine most likely due to the poor oxidation power of this photocatalyst $\left[E_{1 / 2}\left({ }^{*} \mid r^{\prime \prime \prime} / / r^{\prime \prime}\right)=+0.81 \mathrm{~V}\right.$ vs SCE $]$.

[23] RFTA was obtained in one step by acetylation of RF, according to a reported procedure: R. A. Larson, P.L. Stackhouse, T.O. Crowley, Environ. Sci. Technol. 1992, 26, 1792-1798.

[24] C. K. Remucal, K. McNeill, Environ. Sci. Technol. 2011, 45, 5230-5237.

[25] It is reported that electron transfer between ${ }^{3} \mathrm{RFTA}^{*}$ and its ground state can generate the corresponding oxidized and reduced radicals (D-D reaction); see: P. F. Heelis, Chem. Soc. Rev. 1982, 11, 15-39. We acknowledge to one of the reviewers for this suggestion. The [RFTA] ${ }^{-}$is also regenerated after the PET between the ${ }^{3} \mathrm{RFTA}^{*}$ and the carboxylate.

[26] a) For the pKa value of $\mathrm{RFIH} / \mathrm{RFI}^{-}$, see: A. Enrenberg, F. Müller, $\mathrm{P}$. Hemmerich, Eur. J. Biochem., 1967, 2, 286-293; b) For a recent characterization of RFTA where deprotonation of carboxylic acids is proposed by the corresponding radical anion, see: M. März, M. Kohout, T. Neveselý, J. Chudoba, D. Prukata, S. Nizinski, M. Sikorski, G. Burdzinski, R. Cibulka, Org. Biomol. Chem., 2018, 16, 6809-6817.

[27] B. Persson, Acta Chem. Scand. 1977, 31B, 88-89.

[28] Values from $-0.80 \mathrm{~V}$ to $-0.45 \mathrm{~V}$ have been reported, to be dependent on the solvents. See: R. Martinez-Haya, M. A. Miranda, M. L. Marin, Eur. J. Org. Chem. 2017, 2164-2169, and references therein.

[29] The radical generated is stabilized by orbital interactions with both axial ring oxygen lone pair and adjacent $\sigma^{*} \mathrm{C}-\mathrm{O}$ bond (anomeric effect), and reacts from the less hindered convex face. For precedents, see: a) $S$ Ventre, F. R. Petronijevic, D. W. C. MacMillan, J. Am. Chem. Soc. 2015, 137, 5654-5657; b) X. Zhou, P. Wang, L. Zhang, P. Chen, M. Ma, N. Song, S. Ren, M. Li, J. Org. Chem. 2018, 83, 588-603.

[30] Since the reaction mixture is homogeneous during all the process, a possible solution to scale up the reaction would be to use a flow reactor, where the illuminated area-to-reaction volume ratio is maximized. For some recent examples, see: a) J. W. Beatty, J. J. Douglas, R. Miller, R. C. McAtee, K. P. Cole, C. R. J. Stephenson, Chem. 2016, 1, 456-472 b) H. Seo, M. H. Katcher, T. F. Jamison, Nat. Chem. 2017, 9, 453-456 c) F. Lima, L. Grunenberg, H. B. A. Rahman, R. Labes, J. Sedelmeierb S. V. Ley, Chem. Commun., 2018, 54, 5606-5609; d) J. C. Gonzalez Gomez, N. P. Ramirez, T. Lana-Villarreal, P. Bonete, Org. Biomol. Chem. 2017, 15, 9680-9684.

[31] N. P. Ramirez, J. C. Gonzalez-Gomez, Eur. J. Org. Chem. 2017, 2154 2163.

[32] Details can be found in the supporting information file. For a comprehensive discussion of this issue, see: M. A. Cismesia, T. P. Yoon, Chem. Sci. 2015, 6, 5426-5434.

[33] For a recent photocatalytic procedure for the decarboxylative C2 alkylation of benzothiazoles with carboxylic acids, see: B. Wang, P. Li, T. Miao, L. Zou, L. Wang, Org. Biomol. Chem. 2019, 17, 115-122.

[34] The incident light intensity on the reactor was measured using an optical power meter (Thorlabs PM100D) equipped with a thermopile sensor (Thorlabs model S310C).

[35] Prepared according: E. J. Corey, S. Barcza, G. Klotmann, J. Am. Chem. Soc. 1969, 91, 4782 - 4786.

[36] Prepared according to: Sharma, K. K.; Sharma, S.; Kudwal, A.; Jain, R. Org. Biomol. Chem. 2015, 13, 4637 - 4641.

[37] N. N. Bhuvan Kumar, O. A. Mukhina, A. G. Kutateladze, J. Am. Chem Soc. 2013, 135, 9608 - 9611.

[38] The aryl sulfone was only dissolved after few minutes of irradiation.

[39] W. - M. Zao, X. -L. Chen, J. - W. Yuan, L. - B. Qu, L. - K. Duan, Y. - F. Zhao, Chem. Commun. 2014, 50, 2018 - 2020.

[40] M. Eh, Synthesis 2003, 3, 365 - 370. 
WILEY-VCH

\section{FULL PAPER}

\section{Entry for the Table of Contents}

\section{FULL PAPER}

The direct decarboxylative allylation of carboxylic acids with allylsulfones can be efficiently promoted at room temperature by visible light using inexpensive RFTA as photocatalyst in the absence of base. Under similar conditions, some carboxylic acids can be successfully used in the decarboxylative homolytic aromatic substitution of 2(phenylsulfonyl)benzothiazole.

${ }^{\star}$ Riboflavin photocatalysis

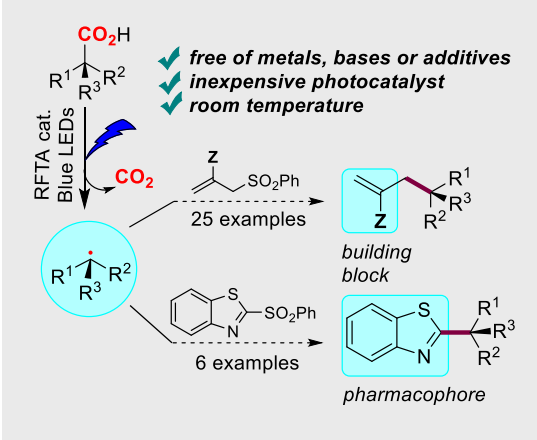

\section{Synthetic Methods*}

Nieves P. Ramirez, Teresa LanaVillarreal, Jose C. Gonzalez-Gomez

Page No. - Page No.

Direct decarboxylative allylation and arylation of aliphatic carboxylic acids using flavin mediated photoredox catalysis 